\title{
Measuring temporal, spectral and spatial changes in electrophysiological brain network connectivity
}

Matthew J. Brookes ${ }^{1}$, George C. O'Neill ${ }^{1}$, Emma L. Hall ${ }^{1}$, Mark W. Woolrich ${ }^{2,3}$, Adam Baker ${ }^{2}$, Sofia Palazzo-Corner ${ }^{1}$, Siân E. Robson ${ }^{1}$, Peter G. Morris ${ }^{1}$ and Gareth R. Barnes ${ }^{4}$

\footnotetext{
${ }^{1}$ Sir Peter Mansfield Magnetic Resonance Centre, School of Physics and Astronomy, University of Nottingham, University Park, Nottingham, UK.

${ }^{2}$ Oxford Centre for Human Brain Activity, University of Oxford, Warneford Hospital, Oxford, UK.

${ }^{3}$ Oxford Centre for Functional MRI of the Brain, University of Oxford, John Radcliffe Hospital, Oxford, UK.

${ }^{4}$ Wellcome Trust Centre for Neuroimaging, University College London, London, UK.
}

\section{KEYWORDS}

MEG; Functional Connectivity; Neural Oscillations; non-stationarity; brain networks; Canonical correlation; multi-variate; leakage reduction

Correspondence to:

Dr. M. J. Brookes,

Sir Peter Mansfield Magnetic Resonance Centre,

School of Physics and Astronomy,

University of Nottingham,

University Park,

Nottingham. NG7 2RD.

E-mail: matthew.brookes@nottingham.ac.uk

RUNNING TITLE

Network non-stationarity

MANUSCRIPT INFORMATION

43 pages; 10 figures; 14,486 words; 


\section{ABSTRACT:}

The topic of functional connectivity in neuroimaging is expanding rapidly and many studies now focus on coupling between spatially separate brain regions. These studies show that a relatively small number of large scale networks exist within the brain, and that healthy function of these networks is disrupted in many clinical populations. To date, the vast majority of studies probing connectivity employ techniques that compute time averaged correlation over several minutes, and between specific pre-defined brain locations. However, increasing evidence suggests that functional connectivity is non-stationary in time. Further, electrophysiological measurements show that connectivity is dependent on the frequency band of neural oscillations. It is also conceivable that networks exhibit a degree of spatial inhomogeneity, i.e. the large scale networks that we observe may result from the time average of multiple transiently synchronised sub-networks, each with their own spatial signature. This means that the next generation of neuroimaging tools to compute functional connectivity must account for spatial inhomogeneity, spectral non-uniformity and temporal non-stationarity. Here, we present a means to achieve this via application of windowed canonical correlation analysis (CCA) to source space projected MEG data. We describe generation of time-frequency connectivity plots, showing the temporal and spectral distribution of coupling between brain regions. Moreover, CCA over voxels provides a means to assess spatial nonuniformity within short time-frequency windows. The feasibility of this technique is demonstrated in simulation and in a resting state MEG experiment where we elucidate multiple distinct spatiotemporal-spectral modes of covariation between the left and right sensorimotor areas. 


\section{1) INTRODUCTION:}

Traditional analysis of neuroimaging data has focussed on the identification of significant changes in some metric of interest that are time locked to a particular task. Such methodologies usually rely on knowledge of task timing, and in some cases accurate models of the temporal evolution of neuroimaging signals which are then compared to measured data. These techniques have proved effective in highlighting brain regions that are involved in sensory and cognitive tasks. However, the last decade has seen a 'paradigm shift' in functional brain imaging (Raichle, 2009), with traditional analyses increasingly complemented by analysis of functional connectivity (Biswal et al., 1995, Beckmann et al., 2005, Fox et al., 2005, Fox and Raichle, 2007, Deco and Corbetta, 2011 ). Here, researchers seek to elucidate spatial patterns of temporal covariation between brain regions. Significant statistical interdependency (e.g. assessed via temporal correlation (Biswal et al., 1995) or independent component analysis (Beckmann et al., 2005)) between signals originating in two or more spatially separate anatomical regions is usually taken to mean that those regions are 'connected'. Functional magnetic resonance imaging (fMRI) has become the most popular technique for mapping these networks of connectivity and this has led to the exciting discovery of a relatively small number of large scale distributed brain networks (Beckmann et al., 2005). These networks appear to be heterogeneous in function (Deco and Corbetta, 2011 ), with some associated with sensory control (e.g. the sensorimotor network) and others relating to cognition and attention (e.g. the dorsal attention network). Networks have been shown to be highly reproducible across subjects, and observable both in the presence and absence of a task (Smith et al., 2009).

In many studies, the methods used to probe connectivity between regions assess temporal correlation over the duration of the measurement, typically several minutes. This approach necessarily assumes that functional connectivity is stationary in time, over the duration of the experiment, and can be captured entirely by a single value of time averaged correlation. However, over a decade of theoretical (e.g. (Friston, 1997, 2000)), computational (e.g. (Breakspear et al., 2003, Honey et al., 2007, Ghosh et al., 2008, Deco et al., 2009)) and empirical (e.g. (Breakspear et al., 2004, Bassett et al., 2006)) evidence suggests that complex and highly temporally variable neuronal dynamics underlie the coupling observed between spatially separate brain regions. Recent studies have explored the temporal evolution of correlation between regions using neuroimaging data. For example, Chang and Glover (Chang and Glover, 2010) employed fMRI to show that functional connectivity is highly variable over time. Further, using magnetoencephalography (MEG), De Pasquale and colleagues have published multiple papers (de Pasquale et al., 2010, de Pasquale et al., 2012) showing that accounting for temporal non-stationarity aids in the detection of several resting 
state networks, suggesting that networks transiently engage with other networks during periods of high internal correlation, with the default mode network acting as a hub of cross network interaction. Also using MEG, Baker et al. (Baker et al., 2012) show evidence of a bi-state nature to band limited power correlation, with periods of zero functional connectivity interspersed with periods of high transient functional connectivity. These findings imply that assessing temporal variability in functional connectivity may provide valuable insight into the neurophysiology of functional networks.

In addition to non-stationarity in time, functional connectivity (as measured by electrophysiological techniques) has also been shown to vary across frequency bands. For example, band limited amplitude envelope correlation between the left and the right motor cortices is maximised in the alpha and beta bands, with correlation failing to reach significance at low frequency (i.e. 1-8Hz) or high frequency (i.e. $>40 \mathrm{~Hz}$ ) (Brookes et al., 2012b). Indeed this finding has been mirrored by other MEG studies (Hipp et al., 2012), and is in general agreement with findings from simultaneous electroencephalography (EEG) / fMRI. The origins of the instability of functional connectivity across frequency bands is shown, to a degree, in a recent paper (Brookes et al., 2012a) which measured the time-frequency evolution of neural oscillatory amplitude in four nodes of a fronto-parietal network during a cognitive task. Results highlighted that in all four nodes, beta power exhibited a monotonic reduction with increased task difficulty. However, stimulus related increases in theta power within this network were only observable in the frontal regions whilst stimulus related decreases in alpha power were only observable in the parietal nodes. In other words, network connectivity, as determined by electrophysiological techniques, is not only non-stationary in time, but also specific to relatively narrow frequency ranges.

Most studies assess functional connectivity either between two spatially separate point locations (i.e. between two voxels), or between two voxel clusters, with signals averaged across voxels within those clusters. This means that, in the same way that time averaged functional connectivity metrics cannot account for temporal non-stationarity, they also cannot account for spatial inhomogeneity. Taking, for example, the sensori-motor network, it is well known that separate sub-regions within the sensorimotor network are mapped somatotopically (i.e. mapped to separate areas of the body (Sanchez-Panchuelo et al., 2012)). Functional connections may be investigated between any pair of sub-regions within the sensori-motor network, and it is entirely conceivable that temporal nonstationarity between individual voxels, or small clusters, may be (in part) due to spatial inhomogeneity within the network. For example, the two somatotopic regions mapped to the left 
and right index fingers may exhibit a functional connection in time window $A$, and likewise the two regions related to the left and right ring fingers may exhibit a functional connection in time window B. Assessment of connectivity between single voxels may therefore only characterise one temporal aspect of connectivity whilst averaging across voxels in large clusters will necessarily spatially blur these effects, as well as introducing increased noise by averaging voxels that do not exhibit correlation.

The above arguments suggest that the next generation of neuroimaging tools to investigate functional connectivity will require the ability to assess temporal non-stationarity, as well as spectral structure and spatial inhomogeneities within (and across) the observed networks. With this in mind, it is noteworthy that electrophysiological metrics such as MEG have significant advantages over fMRI: increased time resolution offers advantages in characterising temporal non-stationarity whilst the direct nature of MEG allows a non-invasive window on neural oscillations, and therefore spectral structure. In this paper, we introduce a novel technique to characterise functional connectivity, based upon beamforming (Van Veen et al., 1997, Robinson and Vrba, 1998, Gross et al., 2001, Sekihara et al., 2006, Brookes et al., 2008) and canonical correlation analysis (CCA) (Soto et al., 2010, Barnes et al., 2011, Brookes et al., 2012b). We extend work presented in our previous papers (Brookes et al., 2011a, Brookes et al., 2012b, Hall et al., 2013) by developing a method capable of measuring the temporal, spectral and spatial variation in functional connectivity, assessed by band limited envelope correlation. Specifically, we use a sliding window to map temporal non stationarity; temporal filtering to detect frequency specific functional connectivity and, most importantly, we apply the multivariate CCA approach across voxels, to characterise the spatial representation of functional connectivity without the need for single seed voxel assessment or cluster averaging. In what follows, Section 2 presents the theoretical basis of CCA within a beamformer framework. In Section 3 we present simulations to show how CCA can achieve the aims set out above. Section 4 shows application of CCA to real MEG data, examining resting state sensorimotor network connectivity. Finally results are discussed and conclusions drawn in Section 5.

\section{2) THEORY:}

Electrophysiological signals are rich in information and the term 'functional connectivity', loosely defined as a statistical dependency between signals originating from different brain regions, can mean a number of things (see e.g. (Scholvinck et al., 2013)). Throughout the remainder of this manuscript, we use the term functional connectivity to mean temporal correlation between the 
amplitude envelopes of band limited neural oscillations (de Pasquale et al., 2010, Liu et al., 2010, Brookes et al., 2011a, Brookes et al., 2011b, Hipp et al., 2012, Luckhoo et al., 2012, Hall et al., 2013).

\section{1) Source Localisation and Selection of Voxels Clusters:}

Characterisation of functional connectivity between two voxel clusters using MEG data necessarily requires that electrophysiological signals are assessed in source space (i.e. extra-cranial magnetic field data are projected into the brain). There are several advantages of source space projection in connectivity assessment (Schoffelen and Gross, 2009). Firstly results can be overlaid directly onto structural brain images, enabling direct interpretation with respect to underlying anatomy. Secondly, source localisation (via adaptive techniques such as beamforming) reduces artifacts from MEG data (Sekihara et al., 2001, Sekihara et al., 2006), meaning that the signal to noise ratio (SNR) of projected data is higher than the SNR of raw data in channel space. This second point is often overlooked, but of critical importance in this context since artifacts caused by common interference across MEG channels (from e.g. the heart) may generate spurious connectivity measurements (Brookes et al., 2011a).

Here, source space projection is achieved via beamforming (Van Drongelen et al., 1996, Van Veen et al., 1997, Robinson and Vrba, 1998, Gross et al., 2001, Sekihara et al., 2001, Brookes et al., 2008); a popular methodology that has been well characterised in previous papers. Briefly, using a beamformer, an estimate of electrical source strength is made at some predetermined location in the brain, using a weighted sum of MEG sensor measurements. The weighting parameters are derived based on power minimisation; the overall power in the output signal is minimised with a linear constraint that power originating from the predetermined location of interest remains in the output signal. A solution to this problem can be derived analytically (Van Veen et al., 1997), and the weighting parameters are based on the data covariance matrix (calculated over a time-frequency window of interest) and the lead field vector, computed individually for each source space voxel. Note that the lead field vector for a particular voxel contains a model of the magnetic fields that would be measured at each of the MEG sensors, in response to a dipole source of unit amplitude with known location and orientation. Beamforming exhibits good spatial resolution and excellent interference suppression. However, a primary assumption is that spatially separate sources are temporally independent, meaning that sources with high temporal correlation between their raw timecourses are supressed, and often mis-localised. 
In what follows, our aim is to measure connectivity via assessment of the interaction between projected signals within two spatially separate voxel clusters. We shall refer to these as the 'seed' cluster and the 'test' cluster. Voxels were defined at the vertices of a regular $(8 \mathrm{~mm})$ grid spanning these regions. A single current orientation was estimated for each voxel, based on a non-linear search for the orientation of maximum signal to noise ratio; this search was limited to the tangential plane due to the relative insensitivity of MEG to radially oriented currents (Robinson and Vrba, 1998).

Following beamformer projection of MEG data, the electrical source timecourses for all voxels within the seed and test volumes are represented by the projected data matrices $\mathbf{X}$ and $\mathbf{Y}$. $\mathbf{X}$ represents data from the seed cluster and has dimensions $f \Delta \times N_{s}$, where $\Delta$ is the duration of the experiment (in seconds), $f$ is the MEG sampling rate (in $\mathrm{Hz}$ ) and $N_{s}$ is the number of voxels contained within the seed cluster. $\mathbf{Y}$ represents data from the test cluster and is of dimension $f \Delta \times N_{t}$, where $N_{t}$ is the number of voxels contained within the test cluster. All subsequent operations are performed on these two matrices.

\section{2) Reduction of Signal Leakage:}

The most significant problem in source space projected MEG metrics of functional connectivity is signal leakage between voxels. This is a direct result of the ill posed nature of the MEG inverse problem, which means that spatially separate source space measurements are not necessarily independent assessments of electrophysiological activity. This, in turn, means that signals generated at one cortical location can 'leak' into MEG estimated activity at spatially separate locations. More specifically, 'leakage' is a collective term encompassing the spatial spread of sources (e.g. characterised by a point spread function) and spatial mis-localisation of sources (e.g. due to an inaccurate lead field model). This effect has been characterised (for beamforming) in previous work (Brookes et al., 2011a) and has been shown to be highly spatially inhomogeneous, meaning that although voxels in close spatial proximity are more likely to be non-independent, there is not necessarily a monotonic relationship with Euclidean distance between the seed and test locations (or clusters). It is clear that spatial leakage between electrophysiological estimates can cause spuriously high estimates of functional connectivity that are driven entirely by inaccurate data projection.

Previous work has focussed on the effect of leakage on functional connectivity estimates between single voxels. Here however, we aim to probe connectivity between larger cortical volumes 
(clusters). Increasing the size of the brain volumes studied makes the chances of observing signal leakage statistically more likely, and for this reason an effective means to reduce leakage between the data matrices $\mathbf{X}$ and $\mathbf{Y}$ is of key importance. It is well known that leakage gives rise to a zerophase-lag linear interaction between projected signals, this fact has been exploited in previous methods (Nolte et al., 2004, Stam et al., 2007, Brookes et al., 2012b, Hipp et al., 2012) where zerophase-lag interaction is removed prior to connectivity assessment. In this paper we implement a multivariate extension to previous work (see appendix (Brookes et al., 2012b, Hipp et al., 2012)) in which linear regression is employed to supress zero-phase-lag interaction between the seed and test regions. This procedure necessarily assumes: 1 ) instantaneous source mixing; 2 ) that source leakage is equivalent for all frequency bands; 3 ) that source leakage is constant in time and 4) that data are Gaussian distributed (see appendix).

To efficiently remove a linear projection of $\mathbf{X}$ on $\mathbf{Y}$, we first reformulate each matrix into an orthogonal basis set; a condition that is never met in MEG since the columns of $\mathbf{X}$ and $\mathbf{Y}$ comprise timecourses from neighbouring voxels which will always contain similar signals due to the inherent smoothness of beamformer reconstruction (and would lead to inflated degrees of freedom in the subsequent multivariate test). To orthogonalise the columns of $\mathbf{X}$ and $\mathbf{Y}$, we employ a technique based on eigenvalue decomposition. We first compute the covariance matrices of $\mathbf{X}$ and $\mathbf{Y}$ thus:

$$
\begin{aligned}
& \mathbf{C}_{\mathbf{X X}}=\mathbf{X}^{T} \mathbf{X} \\
& \mathbf{C}_{\mathbf{Y Y}}=\mathbf{Y}^{T} \mathbf{Y}
\end{aligned}
$$

These covariance matrices are then reduced to their constituent eigenvectors and eigenvalues thus:

$$
\begin{aligned}
& \mathbf{C}_{\mathbf{X X}}=\mathbf{U}_{\mathbf{X}} \mathbf{S}_{\mathbf{X}} \mathbf{U}_{\mathbf{X}}^{T} \\
& \mathbf{C}_{\mathbf{Y Y}}=\mathbf{U}_{\mathbf{Y}} \mathbf{S}_{\mathbf{Y}} \mathbf{U}_{\mathbf{Y}}^{T}
\end{aligned}
$$

The columns of $\mathbf{U}_{\mathbf{X}}$ and $\mathbf{U}_{\mathbf{Y}}$ represent the eigenvectors of $\mathbf{C}_{\mathbf{X X}}$ and $\mathbf{C}_{\mathbf{Y Y}}$ respectively. $\mathbf{S}_{\mathbf{X}}$ and $\mathbf{S}_{\mathbf{Y}}$ are diagonal matrices whose elements correspond to the eigenvalues of $\mathbf{C}_{\mathbf{X X}}$ and $\mathbf{C}_{\mathbf{Y Y}}$. Having found the eigenvectors, it is possible to construct new, orthogonalised versions of $\mathbf{X}$ and $\mathbf{Y}$ which we term $\mathbf{X}_{\mathbf{o}}$ and $\mathbf{Y}_{\mathbf{0}}$ :

$$
\begin{aligned}
& \mathbf{X}_{\mathbf{o}}=\mathbf{X U}_{\mathbf{X}} \\
& \mathbf{Y}_{\mathbf{o}}=\mathbf{Y U}_{\mathbf{Y}}
\end{aligned}
$$

In principle at this stage we could also choose to reduce the dimensionality of the problem (by keeping fewer columns in $\mathbf{U}_{\mathbf{X}}$ and $\mathbf{U}_{\mathbf{Y}}$ ), but we keep all orthogonal components, since we have a large number of temporal degrees of freedom at our disposal. Having collapsed $\mathbf{X}$ and $\mathbf{Y}$ into a 
set of mutually orthogonal vectors we can now reduce the leakage (modelled as any linear combination of the voxel timecourses in $\mathbf{X}$ ) between the two voxel clusters using a multivariate general linear model, where $\mathbf{Y}_{\mathbf{0}}$ is expressed as a linear combination of the features contained in $\mathbf{X}_{\mathbf{0}}$ thus:

$\mathbf{Y}_{\mathbf{o}}=\mathbf{X}_{\mathbf{o}} \boldsymbol{\beta}_{\mathbf{L}}+\mathbf{Y}_{\mathbf{o c}}$

Here, $\boldsymbol{\beta}_{\mathbf{L}}$ represents the combination of orthogonalised features that best describes linear leakage and can be found using:

$\boldsymbol{\beta}_{\mathbf{L}}=\mathbf{X}_{\mathbf{o}}^{+} \mathbf{Y}_{\mathbf{o}}$

Where $\mathbf{X}_{\mathbf{0}}{ }^{+}$denotes the Moore-Penrose pseudoinverse of $\mathbf{X}_{\mathbf{o}}$. Notice that the 'error' term, $\mathbf{Y}_{\mathbf{o c}}$, in Equation 7 actually represents the corrected data matrix for the test cluster and, following computation of $\boldsymbol{\beta}_{\mathbf{L}}$, can be calculated as $\mathbf{Y}_{\mathbf{o c}}=\mathbf{Y}_{\mathbf{o}}-\mathbf{X}_{\mathbf{o}} \boldsymbol{\beta}_{\mathbf{L}}$. Finally, the corrected signal $\mathbf{Y}_{\mathbf{o c}}$ can be transformed from the orthogonalised signal subspace back to voxel space:

$\mathbf{Y}_{\mathbf{c}}=\mathbf{Y}_{\mathbf{o c}} \mathbf{U}_{\mathbf{Y}}^{T}$

Leakage reduction in this way means that linear (zero-phase-lag) interactions between any linear combination of the columns in $\mathbf{X}$ and $\mathbf{Y}_{\mathbf{c}}$ is supressed. However as in the single voxel approach (Brookes et al., 2012b, Hipp et al., 2012) it should be noted that this comes at the expense of any genuine zero-phase-lag interactions (see appendix for a more detailed analysis).

\section{3) Non-Stationarity and Canonical Correlation Analysis:}

Having applied leakage reduction between voxel clusters, we now aim to probe the existence of a statistical interdependency between the envelope voxel timecourses from the seed cluster $\mathbf{X}$, and the (leakage reduced) envelope voxel timecourses from the test cluster $\mathbf{Y}_{\mathbf{c}}$. To compute the envelopes, the individual columns of $\mathbf{X}$ and $\mathbf{Y}_{\mathbf{c}}$ (i.e. the raw voxel timecourses) are Hilbert transformed to obtain the analytic signal; the absolute value of this analytic signal is then computed yielding two new matrices, $\mathbf{E}_{\mathbf{X}}$ (dimension $f \Delta \times N_{s}$ ) and $\mathbf{E}_{\mathbf{Y}}$ (dimension $f \Delta \times N_{t}$ ) whose columns comprise the band limited amplitude envelope signals in different voxels.

$\mathbf{E}_{\mathbf{X}}$ and $\mathbf{E}_{\mathbf{Y}}$ are representative of the whole experiment, (i.e. they each contain $f \Delta$ rows), however the methodology needs to account for non-stationarity in time. For this reason, we now introduce a sliding window of temporal width $\delta$ (in seconds) which is allowed to move in time, and we only assess temporal correlation between clusters within these windows. This concept is shown 
graphically in Figure 1 where the red dotted lines represent the window boundaries. The windowed seed cluster envelope matrix is denoted as $\mathbf{W}_{\mathbf{X}}$ (which has dimension $f \delta \times N_{s}$ ) and the windowed test cluster envelope matrix as $\mathbf{W}_{\mathbf{Y}}$ (which has dimension $f \delta \times N_{t}$ ). Having selected a window, we test for a relationship between the seed and test clusters using a multivariate general linear model, in exactly the same way as described above (Equation 7). Here however, note that we are testing for a linear relationship between the amplitude envelopes of the signal, and not for a linear zero-timelag relationship between the raw signals.

As with leakage reduction, we first account for the fact that separate columns of $\mathbf{W}_{\mathbf{X}}$ or $\mathbf{W}_{\mathbf{Y}}$ are likely to be correlated; again recall that these columns represent envelope timecourses from reconstructed voxels in close spatial proximity. In order to remove this redundancy, and to constrain the degrees of freedom of our test (which will impact on the length of the time window) we decompose these data in a fixed number $(d)$ of orthogonal spatial modes. There are multiple methodologies to impose orthogonality and here eigenvalue decomposition was employed. The covariance matrices for $\mathbf{W}_{\mathbf{X}}$ and $\mathbf{W}_{\mathbf{Y}}$ were computed as:

$$
\begin{aligned}
& \mathbf{W}_{\mathbf{X}}^{T} \mathbf{W}_{\mathbf{X}}=\mathbf{V}_{\mathbf{X}} \mathbf{T}_{\mathbf{X}} \mathbf{V}_{\mathbf{X}}^{T} \\
& \mathbf{W}_{\mathbf{Y}}^{T} \mathbf{W}_{\mathbf{Y}}=\mathbf{V}_{\mathbf{Y}} \mathbf{T}_{\mathbf{Y}} \mathbf{V}_{\mathbf{Y}}^{T}
\end{aligned}
$$

The columns of $\mathbf{V}_{\mathbf{X}}$ and $\mathbf{V}_{\mathbf{Y}}$, which represent the eigenvectors of the covariance of $\mathbf{W}_{\mathbf{X}}$ and $\mathbf{W}_{\mathbf{Y}}$ respectively, were then truncated, leaving only $d$ eigenmodes. Following this, two new matrices are constructed such that:

$$
\begin{aligned}
& \mathbf{W}_{\mathbf{X o}}=\mathbf{W}_{\mathbf{X}} \mathbf{V}_{\mathbf{X T}} \\
& \mathbf{W}_{\mathbf{Y o}}=\mathbf{W}_{\mathbf{Y}} \mathbf{V}_{\mathbf{Y T}}
\end{aligned}
$$

Where $\mathbf{W}_{\mathbf{X o}}$ and $\mathbf{W}_{\mathbf{Y}}$ have $d$ columns and $f \delta$ rows. It is important to note here that at least $4 d$ independent temporal observations are required for the multivariate test to be reliable; and this sets the trade-off between the number of spatial features examined and window length $(\delta)$. The orthogonal nature of the columns in $\mathbf{W}_{\mathbf{X o}}$ and $\mathbf{W}_{\mathbf{Y o}}$ facilitates unambiguous application of the multivariate GLM such that:

$$
\mathbf{W}_{\mathrm{Yo}}=\mathbf{W}_{\mathrm{Xo}} \boldsymbol{\beta}+\boldsymbol{\varepsilon}
$$

Where $\boldsymbol{\beta}$ is the matrix of regression coefficients best predicting $\mathbf{W}_{\mathbf{Y o}}$ from $\mathbf{W}_{\mathbf{X o}}$. This procedure is depicted graphically in Figure 1. 


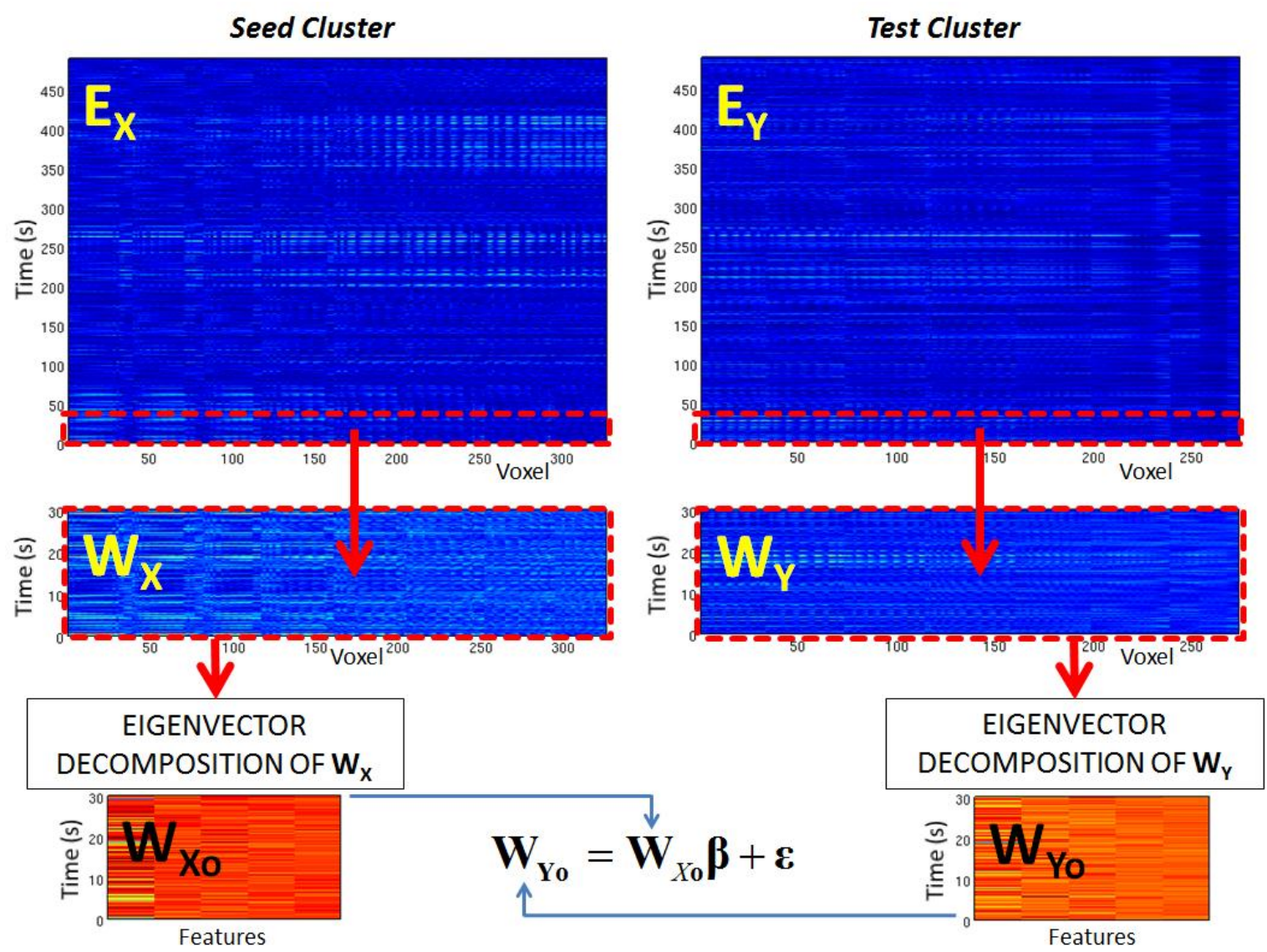

Figure 1: Schematic diagram of the windowed multivariate GLM to test for temporal correlation between band limited amplitude envelopes. The time window, represented by the red dashed lines, allows us to measure functional connectivity as a function of time.

Following computation of $\boldsymbol{\beta}$, it is possible to apply previously established CCA methods (Soto et al., 2009, Soto et al., 2010, Barnes et al., 2011, Brookes et al., 2012b). We first compute the covariance explained by the estimate $\mathbf{W}_{\mathbf{x o}} \boldsymbol{\beta}$ as:

$$
\mathbf{H}=\left(\mathbf{W}_{\mathbf{X o}} \boldsymbol{\beta}\right)^{T}\left(\mathbf{W}_{\mathbf{X o}} \boldsymbol{\beta}\right)
$$

In addition, one can compute the unexplained covariance as:

$$
\mathbf{R}=\left(\mathbf{W}_{\mathbf{Y o}}-\mathbf{W}_{\mathbf{X o}} \boldsymbol{\beta}\right)^{T}\left(\mathbf{W}_{\mathbf{Y o}}-\mathbf{W}_{\mathbf{X o}} \boldsymbol{\beta}\right)
$$

It then becomes possible to compute the matrix

$$
\mathbf{D}=\mathbf{R}^{-1} \mathbf{H}
$$

which corresponds to the ratio of the explained covariance to unexplained covariance. In a univariate sense, this is equivalent to an F-statistic. In the multivariate case, the eigenvalues, $\mathbf{S}_{\mathbf{D}}$, and the associated eigenvectors, $\mathbf{A}$, of $\mathbf{D}$ are defined thus:

$$
\mathbf{D}=\mathbf{A S}_{\mathbf{D}} \mathbf{A}^{-1}
$$


The individual columns of $\mathbf{A}$ (i.e. the eigenvectors) are known as the canonical vectors in $\mathbf{W}_{\mathbf{X o}}$ and show explicitly how to combine the individual orthogonal columns of $\mathbf{W}_{\mathbf{x} \mathbf{0}}$ to best explain the variance observed within and across the columns of $\mathbf{W}_{\mathbf{Y o}}$. In a similar way the canonical vectors in $\mathbf{W}_{\mathbf{Y o}}$ can be computed as:

$\mathbf{B}=\boldsymbol{\beta A}$

The canonical vectors $\mathbf{A}$ and $\mathbf{B}$ can be used to calculate the canonical variates; these comprise the composite timecourses; that is to say the weighted sum of the columns of $\mathbf{W}_{\mathbf{X o}}$ and $\mathbf{W}_{\mathbf{Y o}}$ that maximise temporal correlation, in the window of interest, between the seed and test clusters. The canonical variates in $\mathbf{W}_{\mathbf{X o}}$ are given by $\mathbf{W}_{\mathbf{X o}} \mathbf{B}$ and the canonical variates in $\mathbf{W}_{\mathbf{Y o}}$ are given by $\mathbf{W}_{\mathbf{Y o}} \mathbf{A}$. It then becomes possible to compute the canonical correlation coefficients thus:

$\mathbf{r}_{\mathbf{c a n}}=\left[\left(\mathbf{W}_{\mathbf{X o}} \mathbf{B}\right)^{T}\left(\mathbf{W}_{\mathbf{Y o}} \mathbf{A}\right)\right]\left[\sqrt{\left(\mathbf{W}_{\mathbf{X o}} \mathbf{B}\right)^{T}\left(\mathbf{W}_{\mathbf{X o}} \mathbf{B}\right)\left(\mathbf{W}_{\mathbf{Y o}} \mathbf{A}\right)^{T}\left(\mathbf{W}_{\mathbf{Y o}} \mathbf{A}\right)}\right]^{-1}$

(Note that the square root represents an element by element square root.) The matrix $\mathbf{r}_{\text {can }}$ has dimension $d \times d$ and the elements represent correlation coefficients between the various eigenmodes of correlation. As the eigenmodes are, by definition, orthogonal all off-diagonal elements in this matrix are zero and the diagonal elements represent a single canonical correlation coefficient per eigenmode. For the majority of this paper we focus on the first eigenmode (in which most of the variance is explained), but there is no reason why other modes could not be examined (given that the first mode is significant, see figure 6).

Finally, the canonical vectors can be projected back onto the individual voxels within the seed and test locations. This generates images showing the optimal weighted sum of voxels in the seed cluster that maximally correlate with the optimal weighted sum of voxels in the test cluster. The voxel weightings in the seed location are given by:

$\mathbf{I}_{\mathbf{w X}}=\mathbf{A} \mathbf{V}_{\mathbf{X}}^{T}$

Likewise the voxel weightings in the test cluster are given by:

$\mathbf{I}_{\mathbf{W Y}}=\mathbf{B V}_{\mathbf{Y}}^{T}$

The above theoretical treatment of beamformer projected MEG data allows for computation of the canonical correlation coefficient within each time window, along with images, $\mathbf{I}_{\mathbf{w X}}$ and $\mathbf{I}_{\mathbf{W Y}}$ which describe the combination of voxels which maximise that correlation. Letting the window shift in time facilitates assessment of temporal and spatial structure in correlation. Finally, sequential application to multiple frequency bands enables measurement of the spectral signature of correlation. 


\section{4) Statistical testing via phase randomisation:}

Application of windowed CCA requires careful statistical testing since spurious changes in the temporal profile of correlation can be generated simply as a result of changes in the Fourier components contained within the envelope signals. For example, consider two separate time windows, $A$ and $B$; in time window $A$ the windowed envelope signals $\mathbf{W}_{\mathbf{X}}$ and $\mathbf{W}_{\mathbf{Y}}$ contain correlated Gaussian noise (i.e. exhibit an even distribution across all Fourier components), whereas in time window $B$ those envelope data become coloured (i.e. dominated by a small number of Fourier components). In such a case, the number of temporal degrees of freedom in the data is reduced, and the value of the canonical correlation coefficients $\mathbf{r}_{\text {can }}$ will necessarily increase. This increase is due entirely to the change in spectral structure of the signals and does not represent a genuine change in functional connectivity between the two clusters. Put another way, the background temporal structure in the envelope data will yield non-zero source space correlations that will fluctuate significantly, even if all parameters relating to functional connectivity itself are stationary. For this reason, a robust and reliable statistical technique to account for these 'trivial' changes in functional connectivity must be employed.

The technique used here involves generating surrogate envelope data based upon a phase randomisation process; the reader should note that this theory has been well described elsewhere (Prichard, 1994) and is reviewed here for completeness. For univariate data, phase randomisation is a simple procedure in which, given a univariate time series, $w(t)$, we first compute its discrete Fourier transform $F(w(t))=A(f) e^{i \phi(f)}$ where $F$ denotes a Fourier transform, $A(f)$ is the amplitude of each Fourier component and $\phi(f)$ is the phase. A phase randomised signal, $\widetilde{w}(t)$ can then be generated by rotation of the phase of each Fourier component by a random angle, $\xi(f)$, which is chosen uniformly in the range $0<\xi<2 \pi$ (note that $\xi(f)$ differs for each rotated Fourier component). Mathematically, the phase randomised signal is then given as:

$\tilde{w}(t)=F^{-1}\left[A(f) e^{i(\phi(f)+\xi(f))}\right]$

Note that $\tilde{w}(t)$ has the desirable property that the magnitude of all of the Fourier components (i.e. the power spectrum) is the same as for the original data, and by the Wiener-Khintchine theorem (Prichard, 1994) so is the autocorrelation function.

Equation [23] describes a univariate case, however $\mathbf{W}_{\mathbf{X}}$ and $\mathbf{W}_{\mathbf{Y}}$ are multivariate measurements. In the multivariate case, we not only wish to preserve the Fourier properties of a timeseries, but also the linear correlations between the columns of both $\mathbf{W}_{\mathbf{X}}$ and $\mathbf{W}_{\mathbf{Y}}$; mathematically, we wish to 
preserve the structure of the covariance matrices $\mathbf{W}_{\mathbf{X}}^{T} \mathbf{W}_{\mathbf{X}}$ and $\mathbf{W}_{\mathbf{Y}}^{T} \mathbf{W}_{\mathbf{Y}}$. This can also be achieved via phase randomisation, if the same random sequence $\xi(f)$ is added to each Fourier transformed timecourse (i.e. each Fourier transformed column of $\mathbf{W}_{\mathbf{X}}$ and $\mathbf{W}_{\mathbf{Y}}$ ). Mathematically:

$\tilde{w}_{j}(t)=F^{-1}\left[F\left(w_{j}(t)\right) e^{i \xi(f)}\right]$

where $w_{j}(t)$ represents the $\mathrm{j}^{\text {th }}$ column of $\mathbf{W}_{\mathbf{X}}$ or $\mathbf{W}_{\mathbf{Y}} ; \tilde{w}_{j}(t)$ represents the equivalent $\mathrm{j}^{\text {th }}$ column of a surrogate matrix, which we term $\tilde{\mathbf{W}}_{\mathbf{X}}$ or $\tilde{\mathbf{W}}_{\mathbf{Y}}$. Note that, when constructed in this way, $\tilde{\mathbf{W}}_{\mathbf{X}}$ and $\tilde{\mathbf{W}}_{\mathbf{Y}}$ each individually contain the same power spectra and cross correlation structure as $\mathbf{W}_{\mathbf{X}}$ and $\mathbf{W}_{\mathbf{Y}}$ respectively. However, the phase randomisation means that there should be no correlation between $\tilde{\mathbf{W}}_{\mathbf{X}}$ or $\tilde{\mathbf{W}}_{\mathbf{Y}}$. This being the case, iterative construction of successive realisations of $\tilde{\mathbf{W}}_{\mathbf{X}}$ and $\tilde{\mathbf{W}}_{\mathbf{Y}}$ allow for generation of a null distribution, independently for each time window considered by the windowed CCA. This, in turn, allows for the generation of a dynamic statistical threshold, formed independently for each time window, which accounts for trivial correlations caused by changes in the Fourier components of the envelope signals.

\section{3) SIMULATIONS:}

The theoretical analyses described above were applied in simulation to test the applicability of the technique. All simulations were based on the geometry and data collection parameters of the third order synthetic gradiometer configuration of a 275 channel CTF whole head MEG system (MISL, Coquitlam, Canada) with $5 \mathrm{~cm}$ baseline axial gradiometers. The brain anatomy and head location were based on a real experimental recording session and the simulated sampling rate was $600 \mathrm{~Hz}$. In all cases a multiple local sphere volume conductor head model (Huang, 1999) was employed and the forward solution was based on the dipole model derived by Sarvas (Sarvas, 1987).

\section{1) Null simulation and leakage reduction:}

\section{1a) Methodology:}

The purpose of our first simulation was to assess the performance of CCA, with and without multivariate leakage reduction as described in Section 2.2. In order to test the effectiveness of leakage reduction, null data were simulated. Six spatially separate sources were generated with dipoles located approximately along the motor strip; these locations are shown by the blue overlay in Figure 2. For all six dipoles, the dipolar orientation was tangential to the global sphere radius (computed relative to the mean of all of the local spheres) but randomised with respect to the azimuthal direction. The source timecourses were generated as phase randomised versions of 
genuine (MEG measured) electrophysiological signals (490s in duration), which were estimated from the motor cortex of a single individual during a resting state experiment. Univariate phase randomisation, as described by Equation 23, was applied in order to maintain the measured power spectral distribution of the neural oscillatory signal, whilst destroying any genuine correlation that might exist between the neural signals used. In this way, no interaction was expected between any of the six simulated sources, meaning that if significant interactions were observed they were entirely spurious and likely due to signal leakage. Signals were frequency filtered to the beta band and all sources were given an amplitude of $3 \mathrm{nAm}$. Note that beta oscillations were used since previous work has shown that the strongest interactions between the left and right sensorimotor areas occur in this frequency band (Brookes et al., 2011a). The simulated dipole timecourses were projected through forward solutions for each dipole location/orientation and summed, yielding a simulated sensor space signal matrix. Additive noise data were generated by experimental recording. A 490s MEG recording was made using the third order synthetic gradiometer configuration of a 275 channel CTF MEG system at a sampling rate of $600 \mathrm{~Hz}$, with no subject in the scanner. These 'empty room' data formed the noise matrix which was added to the signal matrix thus generating a simulated MEG data set. The signal to noise ratio, defined as the ratio of the Frobenius norm of the signal matrix to the Frobenius norm of the noise matrix, was calculated as 1.6.

Having simulated MEG data, the beamformer and CCA techniques were applied as described in Section 2 and summarised in Figure 3. Beamformer projected timecourses were reconstructed on an $8 \mathrm{~mm}$ grid within regions of interest covering the bilateral sensorimotor cortices. Those regions of interest are shown by the green overlay in Figure 2 and contained all six simulated sources. The seed cluster (containing 327 voxels) covered approximately the left motor strip and the test cluster (containing 274 voxels) covered approximately the right motor strip. Sliding window CCA was applied to source projected data in the beta band only, with a window width $(\delta)$ of 30 s. The window was allowed to shift in time by $\delta t=2 s$, giving a total of 230 overlapping windows. The dimensionality $(d)$ of the signals following eigenvalue decomposition of the windowed envelope matrices (i.e. the number of columns in $\mathbf{W}_{\mathbf{X o}}$ and $\mathbf{W}_{\mathbf{Y} \mathbf{0}}$ ) was set to 3 . 


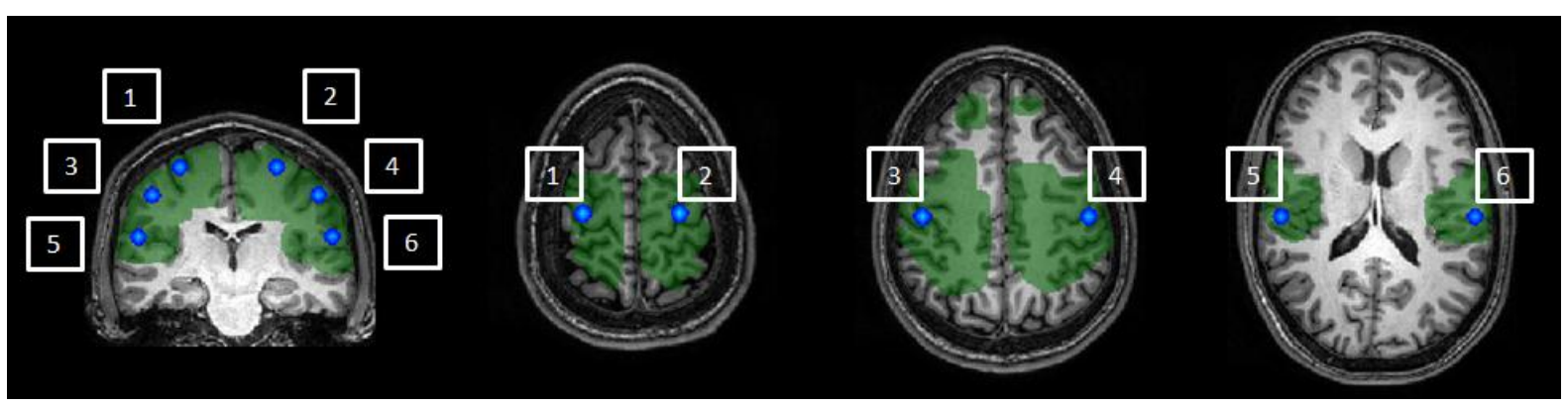

Figure 2: Locations of simulated dipoles in the brain are shown by the blue overlay. The green overlay shows the volume covered by the seed and test voxel clusters.

In order to test the statistical significance of the canonical correlation coefficients computed, multivariate phase randomisation, as described by Equation 24, was employed. For each window, 1000 realisations of the randomised phase matrix $(\xi(f))$ were employed in order to generate surrogate matrices $\tilde{\mathbf{W}}_{\mathbf{X}}$ and $\tilde{\mathbf{W}}_{\mathbf{Y}}$. The CCA technique was then applied to these surrogate matrices in exactly the same way as that used for the real $\mathbf{W}_{\mathbf{X}}$ and $\mathbf{W}_{\mathbf{Y}}$. In this way a null distribution of correlation coefficients was generated independently for each time window. The upper $5^{\text {th }}$ percentile was then computed with Bonferroni correction for multiple comparisons across independent time windows (each window was $30 \mathrm{sec}$ from a total of $490 \mathrm{sec}$ and hence a Bonferroni correction of 490/30 was applied). This was then used as a dynamic statistical threshold. This simulation was repeated with and without signal leakage reduction.

In order to test further the validity of statistical testing via phase randomisation, a second simulation was undertaken. Here the amount by which the window was allowed to shift in time $(\delta t)$ was increased to 30s, meaning that 15 non-overlapping (independent) time windows were employed. The number of iterations of the phase randomisation was reduced to 1 , meaning that a single simulation produced 15 'real' (i.e. based on simulated data) canonical correlation coefficients and 15 surrogate canonical correlation coefficients (based on phase randomised data). This whole processes was repeated 100 times, with the mean and the maximum canonical correlation coefficient, for both real and surrogate data, recorded on each iteration. Once again, this simulation was repeated with and without signal leakage reduction. 


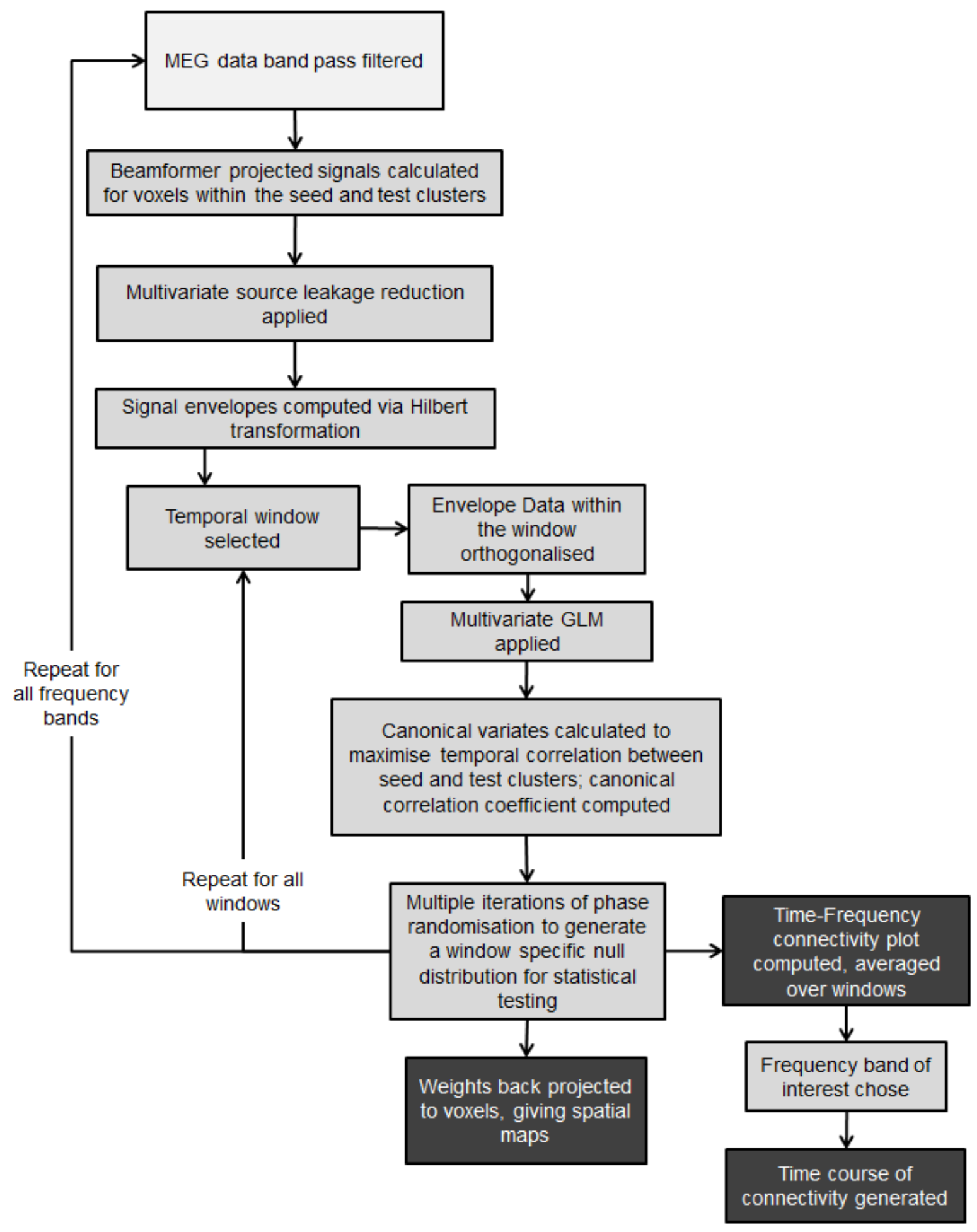

Figure 3: Flowchart summarising the windowed CCA data analysis pipeline. 


\section{1b) Results:}

Figure 4A shows a spatial map, highlighting the effect of leakage reduction on each voxel in the test cluster. The coloured overlay shows the magnitude of the mean square difference between the uncorrected $\mathbf{Y}$ and corrected $\mathbf{Y}_{\mathbf{c}}$ test matrices, plotted across all voxels within the cluster. It is interesting to note that the effects of leakage vary spatially, with the largest effects observed in voxels closest to the seed cluster, as would be expected. Figures $4 B$ and $4 C$ show the timecourse of windowed canonical correlation (blue) for data with (4C) and without (4B) reduction of signal leakage. The dynamic statistical threshold $\left(p_{\text {corrected }}=0.05\right)$, generated by phase randomisation, is shown in red for both cases. Recall that this is a null simulation, with no expected coupling between sources and so the canonical correlation coefficients in the simulated data should remain below the statistical threshold. This is clearly the case for data with leakage reduction, but it is not the case for data without leakage reduction, where (spurious) significant coupling between voxels in the seed and test clusters is induced exclusively as a result of leakage. Figures $4 \mathrm{D}$ and $4 \mathrm{E}$ show histograms of canonical correlation coefficients; histograms in the upper panel were derived using phase randomised (null) data and histograms in the lower panel were derived directly from simulated data. Note that the upper panels in Figures 4D and 4E appear identical as the process of phase randomisation implicitly removes any leakage. Again the effect of leakage reduction is obvious, with no observable difference between histograms in the case where correction is applied.

Finally, Figures $4 \mathrm{~F}$ and $4 \mathrm{G}$ show results of our 100 iteration null simulation, with and without leakage reduction respectively. Bar charts on the left hand side show the mean canonical correlation across 15 non-overlapping windows; bar charts on the right hand side show the maximum canonical correlation over those same 15 windows. Results show mean values across all 100 iterations of the simulation for the simulated data, and for the null distribution; error bars show standard deviation across iterations. Note that without leakage reduction, statistical testing via phase randomisation is clearly invalid since the simulated canonical correlation coefficients are significantly higher than equivalent values in the null distribution; this is driven purely by leakage and would necessarily lead to false positives in functional connectivity measurements. Following leakage reduction however, the simulated canonical correlation coefficients are equal to the equivalent values in the null distribution. This finding implies that the false positive rate will be controlled accurately by the phase randomisation based statistical test, if leakage reduction is applied. (Note that a more detailed mathematical analysis of leakage reduction is given in the appendix.) 


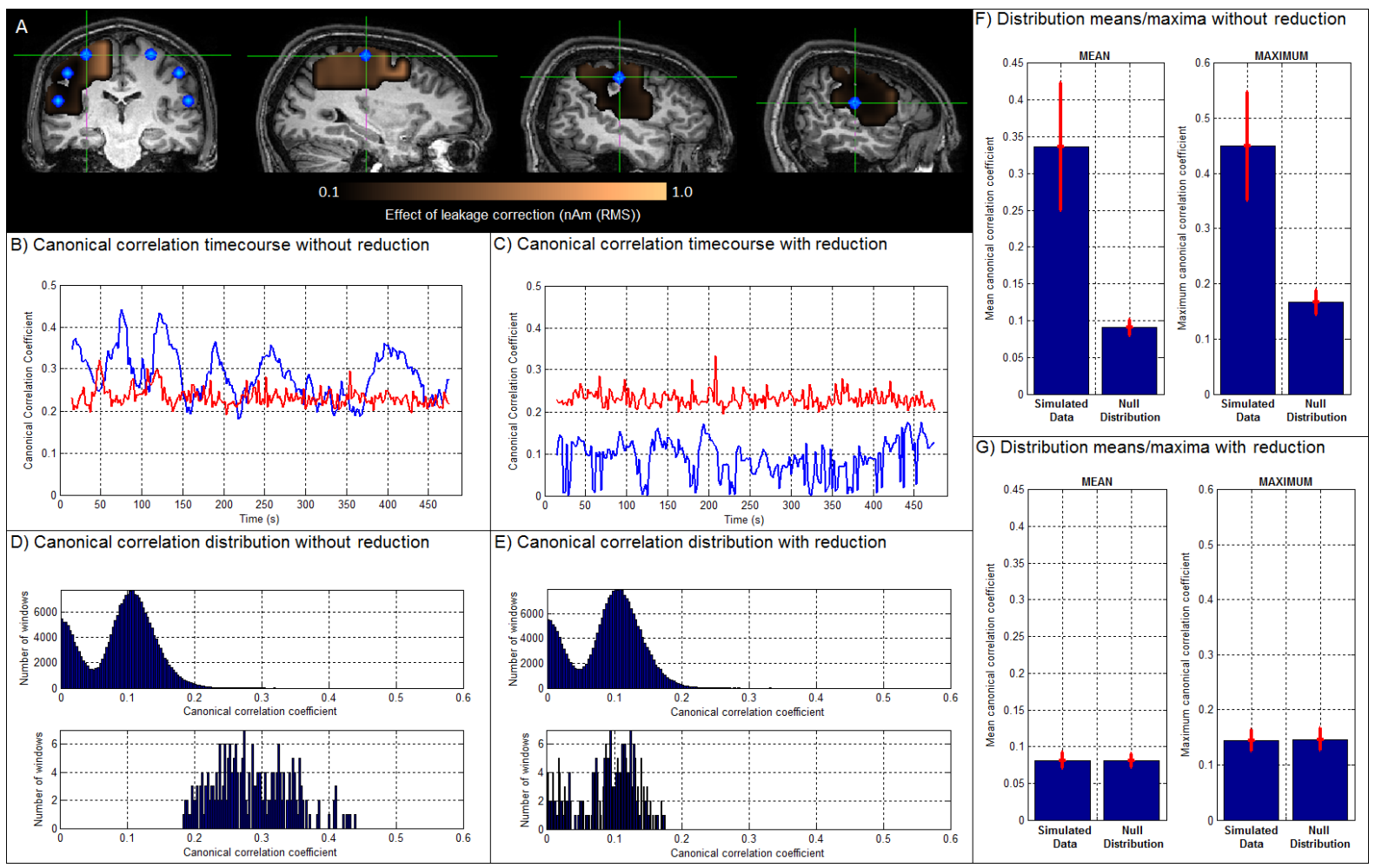

Figure 4: Null simulations and the effect of leakage reduction. A) Spatial map showing the mean effect of leakage reduction on signals at each voxel. The colour overlay represents the mean square difference between the uncorrected $\mathbf{Y}$ and corrected $\mathbf{Y}_{\mathbf{c}}$ matrices, averaged across all time and plotted across voxels; notice that the largest effects of signal leakage are distal to the sources, which are marked by the blue dots. B) and C) show timecourses of canonical correlation for simulated data (blue) and the $p_{\text {corrected }}=0.05$ dynamic statistical threshold (red). The case without leakage reduction is shown in $B$ and with leakage reduction is shown in C. D) and E) show histograms of canonical correlation coefficients. The upper plots show null distributions derived using phase randomisation. The lower plots show distributions from simulated data. Note that without leakage reduction $(D)$ the mean canonical correlation computed using the simulated data is higher than the null distribution; since no temporal correlation has been simulated in this case, this is an example of spurious correlation. Note also that with leakage reduction (E), the canonical correlation for the simulated corrected data is very similar to the null distribution, highlighting the fact that leakage reduction eliminates the spurious correlations shown in (B). F) and G) show mean and maximum canonical correlation coefficients across 100 iterations of the null simulation (error bars show standard deviation). Note again the difference between the cases with (G) and without (F) leakage reduction.

\section{2) Proof of Principle Simulation:}

\section{2a) Method:}

The purpose of the second simulation was to test the beamforming and windowed CCA approach in the case where genuine coupling between dipole timecourses was simulated. Again six spatially separate sources were simulated at the same locations as those employed above (see Figure 2). As previously, all six dipoles were orientated tangential to the radial orientation, with amplitude 3nAm. Source timecourses were again generated as phase randomised versions of genuine (MEG measured) electrophysiological signals (490s in duration), which were estimated from the motor 
cortex of a single individual during a resting state experiment. These were frequency filtered into the $13-30 \mathrm{~Hz}$ band. Temporal correlation between two sources was simulated within specific time windows, via multiplication by a modulatory function. To illustrate this mathematically, consider the case of two sources, labelled $a$ and $b$. To impose coupling, we employ the following formulae:

$s_{a_{-} \text {correlated }}\left(t_{1}<\tau<t_{2}\right)=s_{a_{-} \text {uncorrelaed }}\left(t_{1}<\tau<t_{2}\right) M_{a b}(\tau)$

$s_{b_{-} \text {correlated }}\left(t_{1}<\tau<t_{2}\right)=s_{b_{-} \text {uncorrelaed }}\left(t_{1}<\tau<t_{2}\right) M_{a b}(\tau)$

Here, $s_{a_{-} \text {uncorrelaed }}$ and $s_{b_{-} \text {uncorrelaed }}$ represent the simulated neural signals for sources a and b respectively, in the absence of coupling. The window $\left(t_{1}<\tau<t_{2}\right)$ designates the timing of the transient coupling between a and b. $M_{a b}(\tau)$ is a modulatory function which simulates temporal correlation and $s_{a_{-} \text {correlated }}$ and $s_{b_{-} \text {correlated }}$ represent the transiently coupled timecourses. $M_{a b}(\tau)$ was derived from a real MEG recording, and comprised genuine 70s segments of a beta band amplitude envelope, extracted via beamforming from the motor cortex of a single subject in the resting state (data from (Brookes et al., 2011a)). There were 6 simulated sources (labelled 1-6 in Figure 2); coupling between sources 5 and 2 was simulated in the time window $50 s<t<120$ s; coupling between sources 3 and 4 was simulated in the time window 200s $<t<270 s$; coupling between sources 1 and 6 was simulated in the time window $350 s<t<420$ s. This generated three coupled source pairs defined by three independent modulatory functions $M_{52}(\tau), M_{34}(\tau)$ and $M_{16}(\tau)$. This methodology induces a transient (partial) temporal correlation between the amplitude envelopes of the source pairs, within the time windows specified.

Following dipole timecourse generation, the simulation of MEG data was equivalent to that described in section 3.1a. Timecourses were projected to the MEG sensors using a dipole forward solution and noise data added based on the empty room recording, generating a simulated dataset with SNR of 1.6. CCA was applied as described in section 2, with leakage reduction. 


\section{2b) Results:}

Figure 5 shows results of the proof of principle simulation. Figure $5 \mathrm{~A}$ represents the ground truth: that is, the temporal evolution of coupling between the simulated timecourses. The upper panel shows correlation between sources 5 and 2, the centre panel correlation between sources 3 and 4 , and the lower panel correlation between sources 1 and 6 . Note that the technique described by Equations 25 and 26 only induces a partial correlation between source pairs, with the magnitude of that correlation reaching an average of approximately 0.6 (Pearson correlation coefficient) within the windows of transient coupling. Figure 5B shows the estimated canonical correlation as a function of time. The centre timecourse (blue line) shows the reconstructed temporal evolution of canonical correlation between the seed and test clusters. Note that since all six sources exist within the clusters, correlation between all three coupled source pairs is captured in a single timecourse. The thin black line shows the dynamic statistical threshold $\left(p_{\text {corrected }}=0.05\right)$ and the thick black line shows the mean of the null distribution (generated via phase randomisation) for each time window. Note that all three simulated interactions yield a significant result in the windowed CCA output. Interestingly, the dynamic statistical threshold also shows temporal structure with the mean of the null distribution, and the $p_{\text {corrected }}=0.05$ threshold, changing in time. These changes are driven by temporal structure in the autocorrelation of the envelope timecourses. The spatial maps above and below the timecourse show individual images (derived from $\mathbf{I}_{\mathbf{w X}}$ and $\mathbf{I}_{\mathbf{W Y}}$ ) depicting the spatial signature (canonical vectors) of correlation between the left and right clusters. These spatial maps are shown based on 30s time windows centred at $t=75 \mathrm{~s}, 100 \mathrm{~s}, 150 \mathrm{~s}, 225 \mathrm{~s}, 250 \mathrm{~s}, 300 \mathrm{~s}, 375 \mathrm{~s}$ and 400s. Note that the change in spatial signature as a function of time is in agreement with the simulated connectivity. The blue dots show the locations of the simulated sources.

It should be noted that CCA is a multi-variate methodology and the output for each window is not a single value of canonical correlation, but rather multiple values, each reflecting a separate eigenmode of correlation (the number of modes is given by the minimum rank of $\mathbf{W}_{\mathbf{X o}}, \mathbf{W}_{\mathbf{Y o}}$; in this case both have the same rank $d$ ). In the present simulation we used $d=3$, thus there are three possible canonical modes of correlation. For completeness, Figure 6 shows the timecourse of the first eigenmode (blue line) alongside the timecourses of the second (red) and third (green) eigenmodes of correlation. As we artificially constructed a single spatial mapping between the voxels at any one time we would expect that the correlation between all source pairs is captured in the first eigenmode, with neither the second nor third eigenmodes showing significant deviation from zero. 


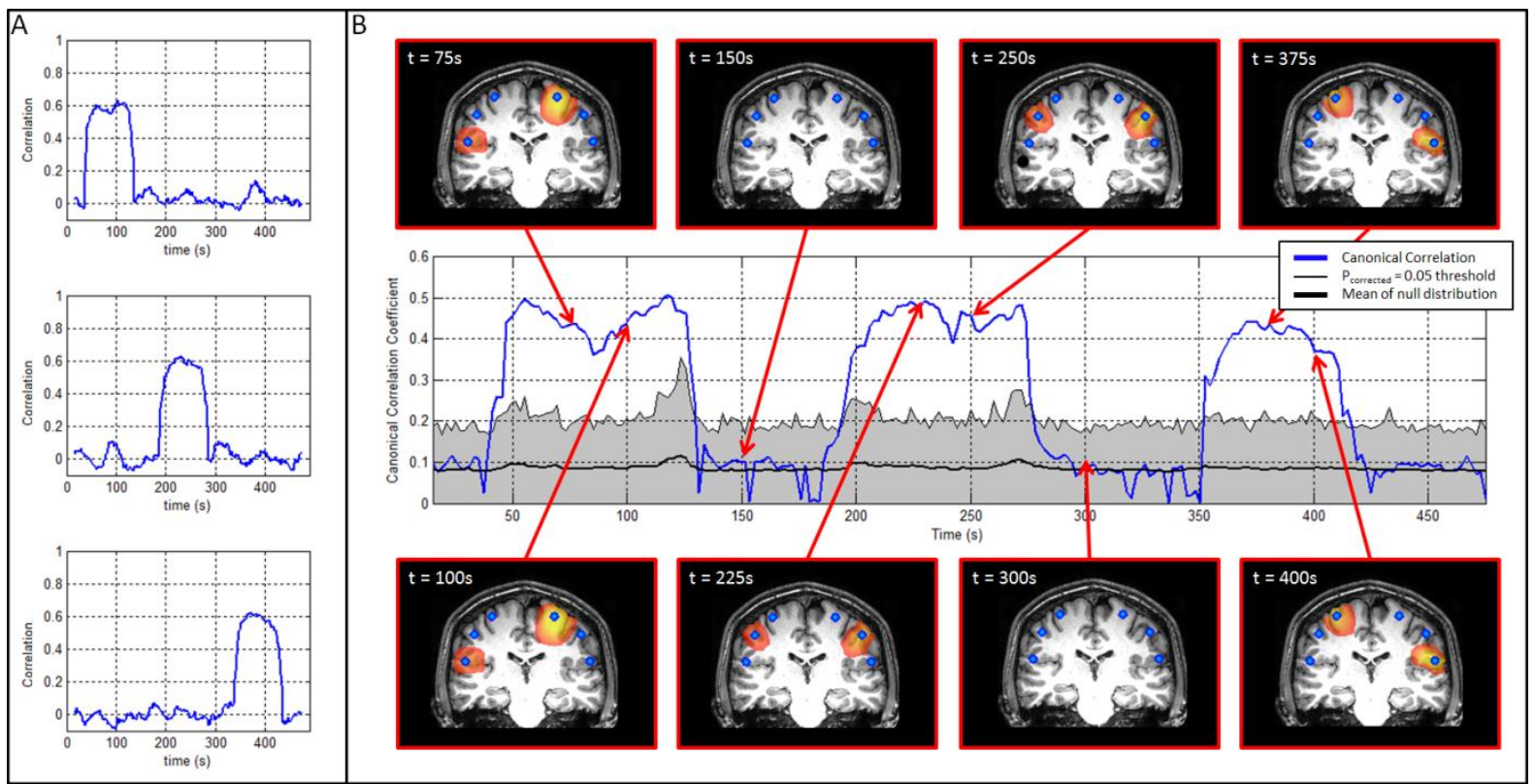

Figure 5: Results of the proof of principle simulation. A) Shows the temporal evolution of simulated connectivity computed using timecourse data. The upper panel shows the timecourse of connectivity between sources 5 and 2; the centre panel shows the timecourse of connectivity between sources 3 and 4; the lower panel shows the timecourse of connectivity between sources 1 and 6. B) Connectivity reconstructed using CCA. The centre timecourse shows the reconstructed temporal evolution of connectivity between the seed and test clusters in the left and right motor strip respectively. Periods of significant temporal correlation are highlighted by the blue line passing outside the shaded region, which is bounded by a $p_{\text {corrected }}=0.05$ statistical threshold derived independently for each window, and corrected for multiple time windows. The thick black line shows the mean canonical correlation for the null distribution, generated via phase randomisation. The spatial maps show individual images (i.e. $\mathbf{I}_{\mathbf{W X}}$ and $\mathbf{I}_{\mathbf{W Y}}$ ) depicting the spatial signature (canonical vectors) of correlation between the left and right clusters. Note the change in spatial signature as a function of time is in agreement with the simulated connectivity. The blue dots show the locations of the simulated sources.

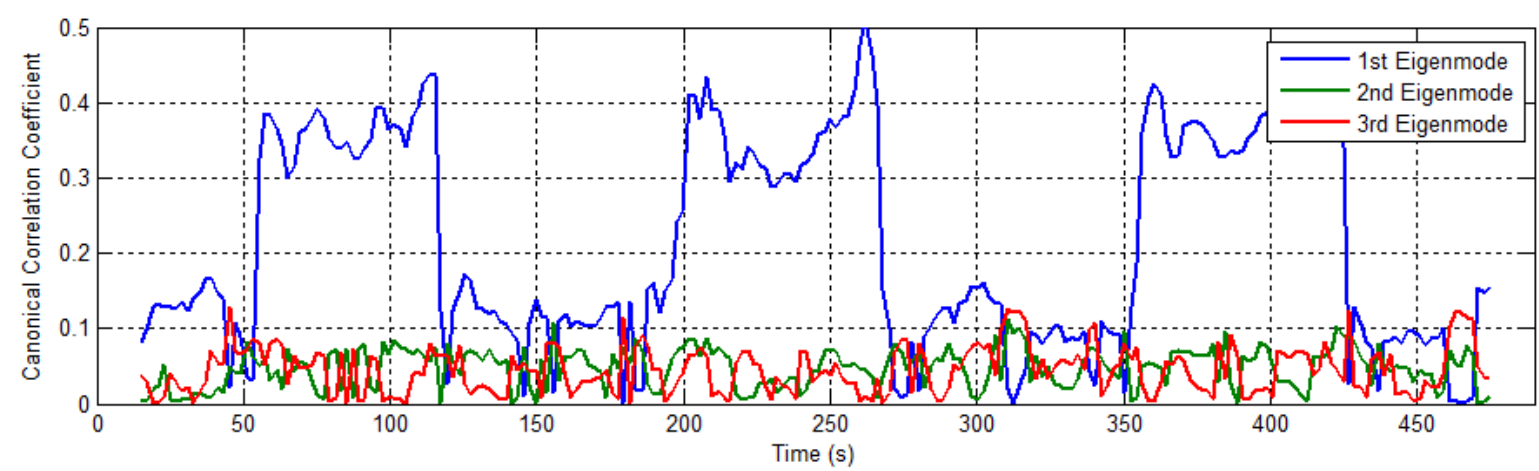

Figure 6: The timecourse of canonical correlation for all three eigenmodes. The blue line shows the first eigenmode which describes all of the simulated amplitude envelope correlation (note this is the same plot as that shown in Figure 5B and is included here again for comparison). The green and red lines show the second and third eigenmodes respectively; note that in this case these higher modes exhibit no significant effect. 


\section{1a) Methodology: data acquisition}

Following application of windowed CCA in simulation, the same technique was applied to real MEG data. Data were acquired at a sampling rate of $600 \mathrm{~Hz}$ using the third order synthetic gradiometer configuration of a 275 channel MEG system (MISL, Coquitlam, Canada) with a $150 \mathrm{~Hz}$ low pass antialiasing filter. Subjects were asked to lie (supine) in the MEG system, with their eyes open and 'rest' whilst 600s of extra-cranial magnetic field data were acquired. Prior to the recording, three localisation coils were attached to the head as fiducial markers (nasion, left preauricular and right preauricular). Energising these coils during data acquisition enabled localisation of the head relative to the MEG sensors. In order to co-register the MEG sensor geometry to the brain anatomy, the subject's head shape was digitised (Polhemus Isotrack) relative to the fiducial markers. MR images were acquired using a 3T Phillips Achieva MR system running an MPRAGE sequence at $1 \times 1 \times 1 \mathrm{~mm}^{3}$ resolution. Coregistration was then achieved by matching the digitised surface to the head surface extracted from the subject's volumetric anatomical MR image. This experimental procedure was approved by the local research ethics committee.

\section{1b) Methodology: data analysis}

The recorded MEG data were inspected visually and segments containing excessive noise removed. These data were then processed using the technique described in Section 2 and summarised in Figure 3. Seed and test clusters were defined covering the left and right sensorimotor areas respectively; these regions are highlighted by the green overlay in Figure 7A. Beamforming was applied in order to reconstruct timecourses of electrical activity on an $8 \mathrm{~mm}$ cubic grid spanning the seed and test clusters. The beamforming and CCA method (Figure 3) was applied iteratively (treating each band independently) over multiple overlapping frequency bands $(4-8 \mathrm{~Hz}, 6-10 \mathrm{~Hz}, 8-13 \mathrm{~Hz}, 10$ $15 \mathrm{~Hz}$ and subsequent overlapping windows ( $10 \mathrm{~Hz}$ bandwidth, $5 \mathrm{~Hz}$ overlap) up to $105 \mathrm{~Hz}$ ). For each band we used a fixed window width $(\delta)$ of 40 s, a total of 280 windows, and a dimensionality (i.e. $d$, the number of columns in $\mathbf{W}_{\mathbf{X o}}$ and $\mathbf{W}_{\mathbf{Y}}$ ) of 3. The values of the canonical correlation coefficients, computed independently for each time window and frequency band, were used to construct a timefrequency (t-f) connectivity plot.

Having computed canonical correlation across all frequencies, a single band of interest was identified for further analysis. MEG data were filtered in the $10-35 \mathrm{~Hz}$ band and again beamforming was applied to reconstruct timecourses on an $8 \mathrm{~mm}$ cubic grid spanning the seed and test clusters. CCA was applied, as described above, and images $\left(\mathbf{I}_{\mathbf{w X}}\right.$ and $\left.\mathbf{I}_{\mathbf{W Y}}\right)$ were computed within each time 
window. For each window, $\mathbf{I}_{\mathbf{w X}}$ and $\mathbf{I}_{\mathbf{W Y}}$ (which represent the seed and test clusters respectively) were combined into a single image, thus generating a total of 280 separate spatial maps, each showing the weightings for voxels (canonical vectors) in the left and right sensorimotor region that describe optimal correlation between clusters. A timecourse of canonical correlation coefficients was also generated, and the significance of each coefficient computed using the phase randomisation approach, with correction for multiple comparisons across independent windows applied using the Bonferroni method. Although separate timecourses and image sets can be computed for each canonical mode, in this example only the dominant mode is considered.

The set of 280 volumetric images (one per time window) show changes in the spatial signature of functional connectivity. However visualisation of this set of images is not trivial. In cases where a task has been employed, one might pick particular time windows that correspond to specific aspects of the task. In the present case however, since the MEG data represent subjects in a 'resting' state, any selection of time windows is somewhat arbitrary. A new set of problems therefore arise - how to identify the number of significantly different canonical vectors or spatial modes. For simplicity we collapsed our 280 images into a smaller number of spatial patterns. To do this, first a covariance matrix was constructed, with dimension $280 \times 280$ whose $\mathrm{ij}^{\text {th }}$ element contained the spatial covariance of image $i$ with image $j$. This matrix was then decomposed into its constituent eigenvectors and eigenvalues. The eigenvectors were multiplied by the images in order to generate volumetric maps showing the spatial signature of each eigenmode; these maps are henceforth termed spatial modes and effectively represent orthogonal spatial patterns of connectivity observed within the 280 image set. The eigenvectors represent the weighting of each individual time window to a particular spatial mode, and can be thought of as a time series showing the contribution of each time point to that mode.

\section{2) Resting state MEG data: Results}

Figure 7 shows the primary results of beamforming and windowed CCA applied to resting state MEG data. Figure 7B shows the t-f connectivity plot, which facilitates visualisation of the temporal and spectral evolution of windowed band limited amplitude envelope correlation between voxel clusters in the left and right sensorimotor regions, in the resting state. Note the high degree of temporal and spectral non-uniformity: The value of canonical correlation exhibits a large variation in time, with high correlation ( 0.6) in some windows and close to zero in other windows. Canonical correlation also exhibits a large degree of variation across frequency with the largest effects observed in the 8- 
$35 \mathrm{~Hz}$ frequency band. This is also evidenced by Figure $7 \mathrm{C}$, which shows the time average of canonical correlation plotted as a function of frequency.

The temporal and spatial variation of connectivity in the $10-35 \mathrm{~Hz}$ frequency band is shown in Figure 8. The centre timecourse (blue line) shows the reconstructed temporal evolution of canonical correlation between the seed and test clusters in left and right sensorimotor cortices respectively. The thin black line shows the dynamic statistical threshold $\left(p_{\text {corrected }}=0.05\right)$ and the thick black line shows the mean of the null distribution (generated via phase randomisation) for each time window. Note that, in agreement with other results (de Pasquale et al., 2010, Baker et al., 2012) there is significant temporal variation in resting state correlation. As with the simulated data, the dynamic statistical threshold and mean canonical correlation calculated for the null distribution shows significant temporal structure. This temporal structure shows that a degree of temporal variability in metrics of functional connectivity can be generated purely as a result of changes in the Fourier component that make up the source timecourses in a given window.

The spatial maps in Figure 8 show coronal and axial aspects of individual images depicting the spatial signature of correlation between clusters. These images are computed within 40 s time windows centred at $t=22 \mathrm{~s}, 80 \mathrm{~s}, 172 \mathrm{~s}, 226 \mathrm{~s}, 294 \mathrm{~s}, 460 \mathrm{~s}, 472 \mathrm{~s}$ and $562 \mathrm{~s}$. The nature of resting state experiments means that these time points are selected somewhat arbitrarily (although all windows correspond to periods of significant temporal correlation). It is interesting to note that, in addition to the temporal and spectral variability shown by Figures 7 and 8 , a degree of spatial inhomogeneity in the network maps exists across separate time windows; and this will be addressed further in the discussion. 


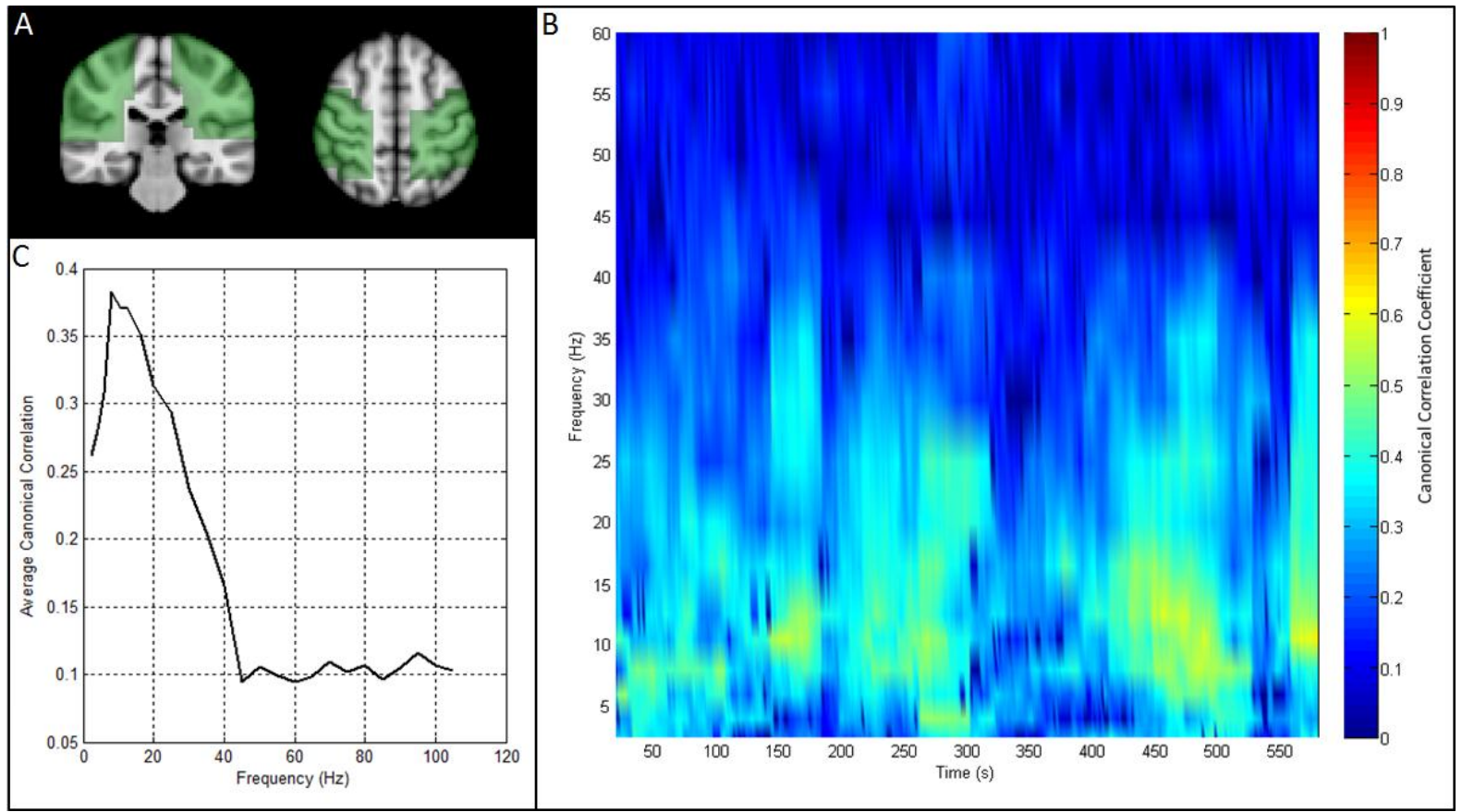

Figure 7: Resting state motor network connectivity. A) Green overlays show the anatomical locations of the seed and test clusters, in left and right sensorimotor regions respectively. B) Time frequency connectivity plot showing the temporal and spectral evolution of band limited amplitude correlation between voxel clusters in the left and right sensorimotor regions. $C$ ) Average connectivity spectrum, showing that the highest average motor network connectivity occurs in the alpha and beta bands.

Finally, Figure 9 shows the separate spatial modes of connectivity computed using eigenvalue decomposition of a matrix of spatial covariance. (NB - spatial modes shown are distinct from the eigenmodes of (CA). The maps in Figure 9A and 9B show the first two spatial modes for a single subject. Note that two separate and distinct spatial patterns are observed. The first shows a symmetric spatial pattern involving bilateral primary sensorimotor cortices, approximately covering the hand area. This pattern has been commonly observed in previous studies. The second spatial mode, whilst again exhibiting symmetry across hemispheres, appears to show effects in inferior axial slices, possibly involving the secondary somatosensory region. Timecourses showing the contribution of each time window to the first and second spatial modes are shown in $9 \mathrm{C}$ and $9 \mathrm{D}$ respectively. For comparison, Figure 9E shows a time average of all 280 images. 


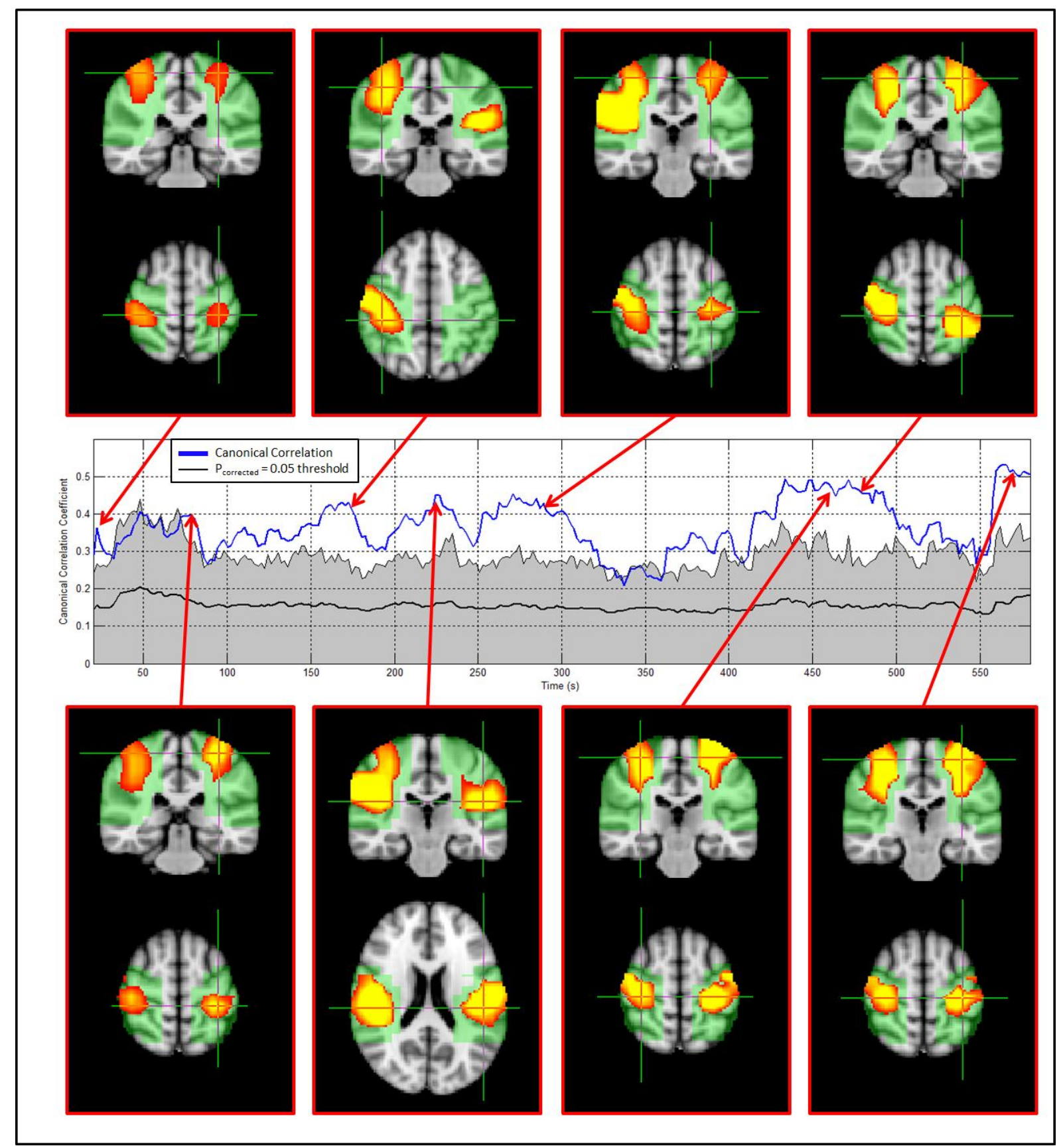

Figure 8: Spatial patterns of connectivity in the $10-35 \mathrm{~Hz}$ frequency band. The centre (blue) timecourse shows the reconstructed temporal evolution of connectivity between the seed and test clusters in the left and right motor strip respectively. Periods of significant temporal correlation are highlighted by the blue line passing outside the shaded region, which is bounded by a $p_{\text {corrected }}=0.05$ statistical threshold derived independently for each window (and corrected for multiple windows). The thick black line shows the mean canonical correlation for the null distribution, generated via phase randomisation. The spatial maps show coronal and axial aspects of the individual images (i.e. $\mathbf{I}_{\mathbf{W X}}$ and $\mathbf{I}_{\mathbf{W Y}}$ ) depicting the spatial signature of correlation between the left and right clusters within 30s time windows centred at selected time points $t=22 \mathrm{~s}, 80 \mathrm{~s}, 172 \mathrm{~s}, 226 \mathrm{~s}, 294 \mathrm{~s}, 460 \mathrm{~s}, 472 \mathrm{~s}$ and $562 \mathrm{~s}$. Note that there is a degree of spatial inhomogeneity over time. 


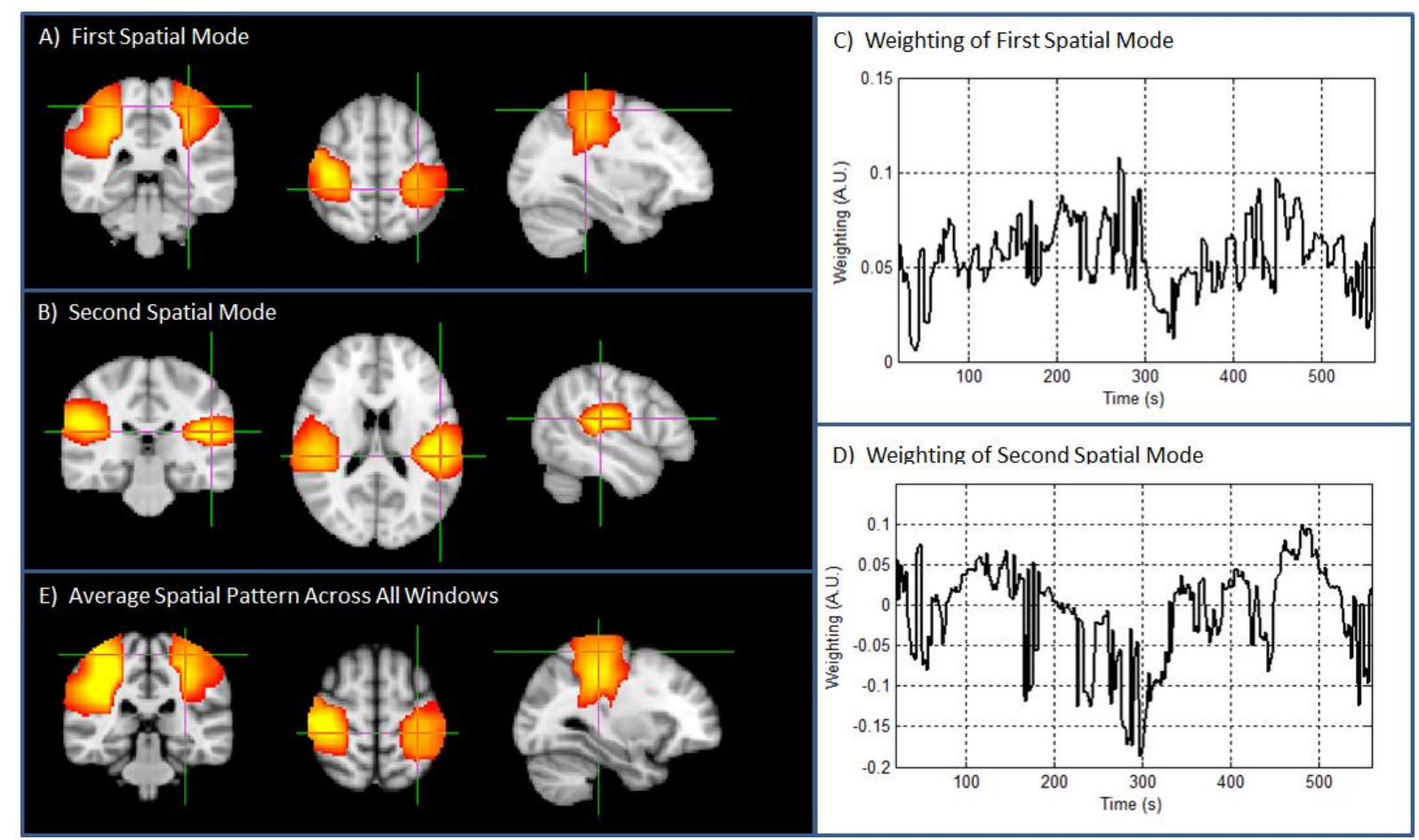

Figure 9: Spatial modes of correlation. A) and B) show the first and second spatial modes of correlation respectively; the timecourses showing the contribution of each time window to the first and second spatial modes are shown in C and D. E) shows the simple time average of all 500 images.

\section{5) DISCUSSION:}

The next generation of tools to compute functional connectivity in neuroimaging data must account for temporal non-stationarity, spatial inhomogeneities, and spectral structure. Here, we have presented a means to achieve this via application of beamforming and windowed CCA to MEG data. We have shown it possible to generate time-frequency connectivity plots showing the temporal and spectral evolution of coupling between brain regions. Moreover, CCA over voxels provides a means to assess spatial inhomogeneity within those short time-frequency windows. We have demonstrated the feasibility of this technique in simulation, and using real MEG data.

MEG has a number of attractive properties for measurement of connectivity. It can assess directly neuro-electrical activity in the brain, and therefore is not confounded by the artifacts caused by haemodynamics that affect fMRI. Such artifacts potentially confound fMRI connectivity metrics in, for example, patients with vascular abnormalities (e.g. stroke). In addition, MEG exhibits excellent temporal resolution, making it preferable to $\mathrm{fMRI}$ for assessment of temporal changes in connectivity. However, the principal limitation of MEG is that spatial resolution is limited by the ill posed inverse problem. In the context of connectivity, this means that spurious measurements of correlation between spatially separate brain regions can be driven exclusively by signal leakage between voxels. In this paper, we extended a previous idea (Brookes et al., 2012b, Hipp et al., 2012) 
for leakage reduction based on removal of linear (zero-phase-lag) interactions between beamformer projected source time series in the seed and test clusters. Source leakage between voxels in MEG is necessarily zero-phase-lag, and removal of this component has been demonstrated by previous papers (Brookes et al., 2012b, Hipp et al., 2012) as an effective means to reduce spurious interactions. Here we extended the regression idea from the univariate case presented previously (Brookes et al., 2012b, Hipp et al., 2012), to a multivariate case. This extension facilitates removal of linear interactions between all voxels (and all linear mixtures of voxels) in the seed and test clusters. As expected, the magnitude of the effect of this reduction differs across voxels within the clusters; this was shown in Figure 4A, with the largest degree of reduction in voxels located in close proximity to the seed cluster. Empirical evidence for the success of this method was given in Figures 4B - 4G. Without leakage reduction, canonical correlation coefficients between the seed and test cluster were higher in the simulation than for a phase randomised case. Recall that phase randomisation not only destroys genuine correlation (i.e. functional connectivity) but also destroys spurious correlation caused by leakage. This means that prior to leakage reduction, a significant difference in canonical correlation between simulated and phase randomised data would be driven entirely by leakage - this was observed in Figures $4 \mathrm{~B}, \mathrm{D}$ and F. Following leakage reduction however, this difference would be expected to be eliminated, and this was indeed evidenced by Figures 4C, E and G. The empirical evidence presented therefore adds weight to previous studies (Brookes et al., 2012b, Hipp et al., 2012) in showing that regression based leakage reduction is effective in ensuring a correct false positive rate in subsequent connectivity assessment (see also appendix).

The windowed CCA approach allows assessment of the temporal evolution of functional connectivity between the seed and test clusters. Furthermore, application within multiple frequency bands enables effective measurement of the spectral signature of temporal correlation. Multiple previous studies (Chang and Glover, 2010, de Pasquale et al., 2010, Brookes et al., 2011a, Baker et al., 2012, de Pasquale et al., 2012) have shown that functional connectivity is dynamic and that temporal correlation between spatially separate brain areas exhibits large changes in time; this observation has been made using both $\mathrm{fMRI}$ and MEG. The results presented in Figures 7 and 8 are in agreement with this, showing large dynamic changes in canonical correlation between the left and right motor clusters. In addition our results show strong frequency dependence with the highest values of temporal correlation observed in the alpha and beta frequency band; this again is in agreement with previous literature (Mantini et al., 2007, Brookes et al., 2011a, Hipp et al., 2012). One of the problems with measurement of temporal correlation in short windows is that of SNR. MEG data exhibit inherently low SNR, and the data captured within the small time-frequency windows used 
here are unaveraged, making accurate measurement of temporal coupling challenging. CCA, applied across voxels, is helpful in this context since is allows a principled way to generate a weighted average of signals across multiple voxels in source space. Averaging voxel timecourses in this way enables an effective increase in the SNR of the data, and hence a more accurate means to assess the time-frequency evolution of connectivity.

Statistical thresholding to define time-frequency windows exhibiting significant temporal correlation is non-trivial. As described in section 2.4, changes in the temporal profile of correlation can be generated simply as a result of changes in the temporal autocorrelation of the envelope time series across multiple time windows. Such temporal structure in the envelope timecourse for the seed and test regions will yield changes in correlation; such changes are trivial, and driven not by a genuine change in functional coupling between regions, but by changes in the Fourier components that make up the signal. In this paper, we apply a previously described technique (Prichard, 1994) to correct for such trivial changes in canonical correlation by employing a dynamic statistical test based on multivariate phase randomisation. By building a null distribution based on Equation 24, we ensure that the canonical correlation coefficients defining that null are constructed using surrogate windowed envelope timecourses with the same autocorrelation function as the real data. This means that any changes in correlation driven purely by changes in signal characteristics are accounted for by the statistical threshold. It is interesting to note that, in real MEG data, this approach yields a dynamic statistical threshold that exhibits marked changes in time. Future work using MEG (or fMRI) to measure dynamic changes in functional connectivity should bear this issue in mind, and consider methods that account for this temporal non-stationarity.

As with all neuroimaging methodologies, windowed CCA requires selection of a parameter set upon which the algorithm is based. The key parameters are 1); the voxel cluster size, 2) the number of eigenmodes (d) retained within each window and 3) the time frequency window size. Judicious selection of regions of interest is key to the CCA technique. If regions are made too small, one loses spatial degrees of freedom and ultimately the CCA technique collapses to univariate correlation. Alternatively, if regions are made to large, one may dilute the effects of interest in specific brain areas, by incorporating other regions which contribute orthogonal signals. Selection of regions of interest, for the present study, was based upon the sensorimotor network previously defined by fMRI (Smith et al., 2012), however it is equally possible to select regions based on cortical parcellation. Ultimately, region selection depends on the precise scientific question to be addressed. Selecting the number of retained eigenmodes, $d$, is linked directly to both the volume encompassed 
by the selected regions (larger regions require increased d) and the spatial resolution of the MEG inverse projection within those regions (higher spatial resolution means more independent signals within a cortical volume, necessitating larger $d$ ). This means that, again, selection of $d$ is specific to the particular study being undertaken; this said an objective means to select $d$ can be derived as the percentage of data variance explained by the eigenmodes retained. Finally, judicious selection of a time frequency window involves a trade-off between temporal/spectral resolution and accuracy. The smaller the time frequency window, the less accurate the estimation of canonical correlation. The window size is also related to the number of selected eigenmodes (d) and, as a rule of thumb, one requires more than $4 d$ independent temporal observations within the window for the multivariate test to be reliable. This imposes a fundamental limit on temporal resolution of any sliding window technique. In task based studies, this poses less of a problem since time windows can be made narrow, and the amount of data within a window effectively increased by concatenation of data segments across task trials. However, in the resting state this is not possible. A powerful and complementary alternative to sliding windows, which has particular application in resting state MEG measurements, is to deploy techniques such as Hidden Markov Models (HMMs), which have been shown to detect short-lived re-occurring states in resting state MEG data, characterised by repeating patterns of covariance over channels (Woolrich et al., 2013). This multivariate approach has, so far, been used to perform temporally adaptive MEG source reconstruction and could be readily extended for use with CCA. In addition to these fundamental parameters, windowed CCA as described is critically dependent on source localisation, in this case using beamforming. Parameter selection and optimised application of beamforming is covered extensively in previous literature and will not be reproduced here. However we do note that windowed CCA may, in principle, by applied in conjunction with any inverse projection technique, with the caveat that different inverse projection algorithms exhibit different signal leakage characteristics and the interaction between inverse projection and leakage reduction should be characterised prior to direct application.

Assessment of spatial inhomogeneity in functional connectivity is important if we are to generate a means to measure the spatial signature of 'sub-networks' within previously characterised large scale distributed networks. The CCA approach, as presented, allows a means to measure changes in the spatial signature of connectivity throughout the experiment. The utility of the method was demonstrated by application to the resting state data in Figures 8 and 9 . These results cannot be over interpreted since, although the spatial patterns elucidated have been shown to be consistent over two individuals (see supplementary material) they may not readily extend to a large group. This said, it is clear from Figure 8 that a degree of spatial inhomogeneity is apparent within the motor 
network, with spatially distinct 'sub-networks' exhibiting significant canonical correlation within temporally separated windows. This result was extended further in Figure 9, with the inclusion of volumetric maps depicting two separate spatial modes of correlation. The first spatial mode resembles strongly a well-known sensorimotor network, which is often observed in both bilateral and unilateral motor paradigms. This comprises bilateral and symmetric regions covering (approximately) the hand areas of left and right sensorimotor cortex. The second spatial mode incorporates bilateral and symmetric cortical regions observed in inferior slices. The inherent smoothness of MEG images necessarily makes unambiguous spatial interpretation of these images challenging, but nevertheless this secondary spatial mode is physiologically plausible, and may incorporate the bilateral secondary somatosensory region. Similar spatial patterns were found in a second individual during a resting state MEG acquisition. Methods to derive robust and regularly occurring spatial patterns of connectivity offer a means to extend the CCA technique from single subject application (as presented) to group study. Techniques such as eigenvalue decomposition (as used here) or alternatively k-means clustering, should allow elucidation of consistent spatial patterns across multiple subjects. Alternatively, it is conceivable that concatenating spatially normalised volumetric images across many subjects may generate large multi-subject datasets amenable to processing with techniques such as spatial ICA, which again may elucidate robust and regularly occurring spatial patterns of functional sub-networks within (for example) the sensorimotor system. Although it remains to be seen whether or not our present findings extend across large groups of subjects, they do present an immediate example of the utility of the windowed CCA approach. Further work might attempt to provide a principled identification of the number of spatial and temporal modes of correlation supported by MEG data.

\section{6) CONCLUSION:}

The results presented in this paper show that a combination of beamforming, multivariate leakage reduction, and windowed CCA is a flexible approach to measure the spatial, spectral and temporal evolution of functional connectivity, assessed by temporal correlation of band limited oscillatory amplitude. The utility of this approach has been shown in simulation, and in real resting state MEG data. The technique may also be readily extended to task based studies in order to compute the dynamics of functional connectivity throughout sensory or cognitive paradigms. The method may be extended to other networks, e.g. the default mode network, where previous literature (de Pasquale et al., 2010, de Pasquale et al., 2012) has shown that non-stationarity may be of great importance. In terms of the methodology, this may also be expanded. Here we focus only on application of CCA across multiple voxels, however this may be further extended to multiple frequency bands within 
each voxel, or multiple current orientations within each voxel. The latter case is of particular interest and has been used successfully in a recent study to explore signal coherence in the dorsal attention network (Marzetti et al., 2013). To summarise, our current knowledge of resting state networks is based on assumptions of stationarity; it is reasonable to assume that these are in turn simply dominant modes of a complex series of interactions which this methodology will allow us to explore. 


\section{7) APPENDIX: LEAKAGE REDUCTION USING LINEAR REGRESSION}

The principal limitation of MEG as a means to measure functional connectivity is signal leakage between spatially separate locations. 'Leakage' is a result of imperfect source localisation which, in turn, results from the ill posed MEG inverse problem. The leakage reduction methodology that we employ is a post-hoc fix to limit the effect of poor source localisation on functional connectivity. The idea is to reduce linear (zero-phase-lag) interactions between beamformer projected source time series in the seed and test clusters. This is achieved using linear regression (Brookes et al., 2012b, Hipp et al., 2012), in which we derive a leakage coefficient $\left(\boldsymbol{\beta}_{\mathbf{L}}\right)$ which can be used subsequently to modify the estimate of electrical activity at the test location. To gain further insight into the leakage reduction methodology it proves instructive to undertake a simple analytical analysis.

\section{1) Analytical analysis}

Consider a simple case with two sources, $\mathbf{q}_{1}$ represents the timecourse from our test location $\left(\mathbf{r}_{1}\right)$ whilst $\mathbf{q}_{2}$ represents the timecourse at the seed location $\left(r_{2}\right)$. Assuming no other electrophysiological sources in the brain, the MEG data are described by:

$\mathbf{m}=\mathbf{l}_{1} \mathbf{q}_{1}+\mathbf{l}_{2} \mathbf{q}_{2}+\mathbf{e}$

Where $\mathbf{l}_{1}$ and $\mathbf{l}_{2}$ represent the lead field vectors for sources $\mathbf{q}_{1}$ and $\mathbf{q}_{2}$ and $\mathbf{e}$ represents sensor level noise. Now assume that we employ a beamformer to reconstruct an estimate of source $\mathbf{q}_{1}$ so that:

$\hat{\mathbf{q}}_{1}=\mathbf{w}_{1}^{T} \mathbf{m}(f)$

Note that the 'hat' notation represents an estimate (i.e. $\hat{\mathbf{q}}_{1}$ is an estimate of the true source timecourse $\mathbf{q}_{1}$ ). $\mathbf{w}_{1}$ represents the beamformer weights for location $\mathbf{r}_{1}$, which are given by

$\mathbf{w}_{1}^{T}=\frac{\mathbf{l}_{1}^{T} \mathbf{C}^{-1}}{\mathbf{l}_{1}^{T} \mathbf{C}^{-1} \mathbf{l}_{1}}$

Where $\mathbf{C}$ is the data covariance matrix. Substituting Equations $A 1$ and $A 3$ into $A 2$, and using the definition of a beamformer unit constraint (i.e. $\mathbf{w}_{1}^{T} \mathbf{l}_{1}=1$ ) we can show that:

$\hat{\mathbf{q}}_{1}=\mathbf{q}_{1}+\frac{\mathbf{l}_{1}^{T} \mathbf{C}^{-1} \mathbf{l}_{2}}{\mathbf{l}_{1}^{T} \mathbf{C}^{-1} \mathbf{l}_{1}} \mathbf{q}_{2}$

So in this case, the magnitude of the leakage from the seed location to the test location is given by:

$a=\mathbf{l}_{1}^{T} \mathbf{C}^{-1} \mathbf{l}_{2}\left(\mathbf{l}_{1}^{T} \mathbf{C}^{-1} \mathbf{l}_{1}\right)^{-1}$

We can now undertake an analytical analysis of leakage reduction via regression. (Note that we do this here for a univariate case, although the same argument extends to the multivariate approach.) To reduce leakage of $\mathbf{q}_{2}$ into $\mathbf{q}_{1}$ we employ Equation 7 (simplified for the univariate case): 
$\hat{\mathbf{q}}_{1}=\beta \hat{\mathbf{q}}_{2}+\hat{\mathbf{q}}_{1 M}$

Where $\hat{\mathbf{q}}_{1 M}$ represents the modified source estimate for $\mathbf{q}_{1}$ following leakage reduction. The 'leakage parameter,' $\beta$, is given by the Moore-Penrose pseudo-inverse of $\mathbf{q}_{2}$, mathematically:

$\beta=\left[\hat{\mathbf{q}}_{2}^{T} \hat{\mathbf{q}}_{2}\right]^{-1} \hat{\mathbf{q}}_{2}^{T} \hat{\mathbf{q}}_{1}$

If we assume that $\hat{\mathbf{q}}_{2}$ is a perfect reconstruction (i.e. $\hat{\mathbf{q}}_{2}=\mathbf{q}_{2}$ ), and substitute equation A4 and A5 into $A 6$ we find that:

$\beta=\left[\mathbf{q}_{2}^{T} \mathbf{q}_{2}\right]^{-1} \mathbf{q}_{2}^{T}\left(\mathbf{q}_{1}+a \mathbf{q}_{2}\right)$

If $\mathbf{q}_{1}$ and $\mathbf{q}_{2}$ are temporally uncorrelated (a condition for beamforming) such that $\mathbf{q}_{1}^{T} \mathbf{q}_{2}=\mathbf{q}_{2}^{T} \mathbf{q}_{1}=0$ (this is the case in the infinite integration limit) then:

$\beta=\left[\mathbf{q}_{2}^{T} \mathbf{q}_{2}\right]^{-1}\left(\mathbf{q}_{2}^{T} \mathbf{q}_{1}+a \mathbf{q}_{2}^{T} \mathbf{q}_{2}\right)=a$

In other words, given uncorrelated sources and perfect reconstruction of the interfering source $\mathbf{q}_{2}$, the leakage parameter $\beta$ is an unbiased estimate of the leakage, $a$. We term this case '1-way leakage', meaning that we get leakage of $\mathbf{q}_{2}$ into $\mathbf{q}_{1}$, but no leakage from $\mathbf{q}_{1}$ into $\mathbf{q}_{2}$. This condition may be met if $\mathbf{q}_{2}$ represented a fundamentally different process. For example, the interfering source, $\mathbf{q}_{2}$, may represent cardiac interference and may be measured using an ECG. In such a case the magnetocardiogram could easily leak into a beamformer projected MEG signal, but it is unlikely that a MEG signal could leak back into the ECG measurement. This is therefore a likely case of 1-wayleakage.

Unfortunately, for measurements of functional connectivity between two brain regions, the assumption that $\hat{\mathbf{q}}_{2}=\mathbf{q}_{2}$ will never be met. This is because $\hat{\mathbf{q}}_{2}$ is a beamformer estimated timecourse and if we observe leakage of the seed source into the test source $\left(\mathbf{q}_{2}\right.$ into $\left.\mathbf{q}_{1}\right)$, we are highly likely to observe leakage of the test source into the seed source $\left(\mathbf{q}_{1}\right.$ into $\left.\mathbf{q}_{2}\right)$. We term this more complex case '2-way-leakage'. By analogy with Equation A4, in the case of 2-way-leakage, the beamformer estimate of the seed source $\mathbf{q}_{2}$ will be given by:

$\hat{\mathbf{q}}_{2}=\mathbf{q}_{2}+\frac{\mathbf{l}_{2}^{T} \mathbf{C}^{-1} \mathbf{l}_{1}}{\mathbf{l}_{2}^{T} \mathbf{C}^{-1} \mathbf{l}_{2}} \mathbf{q}_{1}=\mathbf{q}_{2}+b \mathbf{q}_{1}$

Where $b=\mathbf{l}_{2}^{T} \mathbf{C}^{-1} \mathbf{l}_{1}\left(\mathbf{l}_{2}^{T} \mathbf{C}^{-1} \mathbf{l}_{2}\right)^{-1}$. Substituting Equations A4, A5 and A10 into A7 we get:

$\beta=\left[\left(\mathbf{q}_{2}+b \mathbf{q}_{1}\right)^{T}\left(\mathbf{q}_{2}+b \mathbf{q}_{1}\right)\right]^{-1}\left(\mathbf{q}_{2}+b \mathbf{q}_{1}\right)^{T}\left(\mathbf{q}_{1}+a \mathbf{q}_{2}\right)$

Again assuming that $\mathbf{q}_{1}$ and $\mathbf{q}_{2}$ are temporally uncorrelated we find that:

$\beta=\left[\mathbf{q}_{2}^{T} \mathbf{q}_{2}+b^{2} \mathbf{q}_{1}^{T} \mathbf{q}_{1}\right]^{-1}\left(a \mathbf{q}_{2}^{T} \mathbf{q}_{2}+b \mathbf{q}_{1}^{T} \mathbf{q}_{1}\right)$ 
If $\mathbf{q}^{T} \mathbf{q}=N v^{2}=\sigma$ where $N$ is the number of samples in the time course and $v^{2}$ represents the source variance, then the leakage parameter simplifies to:

$\beta=\frac{a \sigma_{2}+b \sigma_{1}}{\sigma_{2}+b^{2} \sigma_{1}}$

Substituting this back into Equation A6 it becomes possible to derive an Equation for the modified estimated source timecourse $\left(\hat{\mathbf{q}}_{1 M}\right)$ following leakage reduction:

$\hat{\mathbf{q}}_{1 M}=\hat{\mathbf{q}}_{1}-\beta \hat{\mathbf{q}}_{2}=\left(\mathbf{q}_{1}+a \mathbf{q}_{2}\right)-\left[\frac{a \sigma_{2}+b \sigma_{1}}{\sigma_{2}+b^{2} \sigma_{1}}\right]\left(\mathbf{q}_{2}+b \mathbf{q}_{1}\right)$

Which simplifies to:

$\hat{\mathbf{q}}_{1 M}=k\left(\sigma_{2} \mathbf{q}_{1}-\sigma_{1} b \mathbf{q}_{2}\right)$

Where $k$ is a constant $\left(k=(1-a b)\left(\sigma_{2}+b^{2} \sigma_{1}\right)\right)$. Equation A15 reveals the key point that, given the case of 2-way-leakage, our leakage reduction algorithm does not correct for limitations of source estimation. This is shown clearly by Equation A15, where we see that the modified timecourse still contains a component $\left(\mathbf{q}_{2}\right)$ originating from the seed location. Note that this is distinct from the 1way leakage case where a perfect reconstruction of $\mathbf{q}_{1}$ would be achieved. However, it is the metric of functional connectivity between $\hat{\mathbf{q}}_{1 M}$ and $\hat{\mathbf{q}}_{2}$, rather than the source estimates themselves that are of primary interest.

Having obtained an expression for $\hat{\mathbf{q}}_{1 M}$ in the 2-way-leakage case, we can derive the magnitude of the signal leakage between $\hat{\mathbf{q}}_{2}$ and $\hat{\mathbf{q}}_{1 M}$, which for the univariate case is given by the correlation coefficient $(r)$ between $\hat{\mathbf{q}}_{2}$ and $\hat{\mathbf{q}}_{1 M}$ :

$r=\frac{\hat{\mathbf{q}}_{1 M}^{T} \hat{\mathbf{q}}_{2}}{\sqrt{\hat{\mathbf{q}}_{1 M}^{T} \hat{\mathbf{q}}_{1 M}} \sqrt{\hat{\mathbf{q}}_{2}^{T} \hat{\mathbf{q}}_{2}}}=\frac{k}{\sqrt{\hat{\mathbf{q}}_{1 M}^{T} \hat{\mathbf{q}}_{1 M}} \sqrt{\hat{\mathbf{q}}_{2}^{T} \hat{\mathbf{q}}_{2}}}\left(\sigma_{2} \mathbf{q}_{1}-\sigma_{1} b \mathbf{q}_{2}\right)^{T}\left(\mathbf{q}_{2}+b \mathbf{q}_{1}\right)$

It is simple to see that, given $\mathbf{q}_{1}^{T} \mathbf{q}_{2}=\mathbf{q}_{2}^{T} \mathbf{q}_{1}=0$, and $\sigma=\mathbf{q}^{T} \mathbf{q}$, then:

$r=\frac{k}{\sqrt{\hat{\mathbf{q}}_{1 M}^{T} \hat{\mathbf{q}}_{1 M}} \sqrt{\hat{\mathbf{q}}_{2}^{T} \hat{\mathbf{q}}_{2}}}\left(\sigma_{2} \mathbf{q}_{1}^{T} \mathbf{q}_{2}+\sigma_{2} b \mathbf{q}_{1}^{T} \mathbf{q}_{1}-\sigma_{1} b \mathbf{q}_{2}^{T} \mathbf{q}_{2}-\sigma_{1} b^{2} \mathbf{q}_{1}^{T} \mathbf{q}_{2}\right)=0$

Recall that we assume any linear interaction between the seed and the test sources is driven purely by signal leakage, and therefore, following leakage reduction, linear correlation between the two sources should be zero. Equation A17 shows that, given an ideal scenario with two sources, and in the infinite integration limit, the regression technique imposes directly zero correlation between the estimated seed timecourse $\hat{\mathbf{q}}_{2}$, and the estimated and modified test timecourse $\hat{\mathbf{q}}_{1 M}$. (See also supplementary material.) 


\section{2) Gaussian Assumptions:}

As shown above, leakage reduction via regression necessarily sets the linear correlation between $\hat{\mathbf{q}}_{1 M}$ and $\hat{\mathbf{q}}_{2}$ to zero. However, in testing for functional connectivity it is correlation between Hilbert envelopes that is measured. It is therefore important to show that having applied leakage reduction, correlation between the envelopes generates an accurate false positive rate.

Figure A1 shows results of a simple simulation in which 2 source timecourses were simulated as mixtures of two independent timecourses. The first source timecourse $\left(\mathbf{s}_{1}\right)$ was given as:

$\mathbf{s}_{1}=\mathbf{x}_{1}+0.2 \mathbf{x}_{2}$

The second timecourse $\left(\mathbf{s}_{2}\right)$ was given as:

$\mathbf{s}_{2}=\mathbf{x}_{2}-0.2 \mathbf{x}_{1}$

Two separate versions of the simulation were run. In the first case, $\mathbf{x}_{1}$ and $\mathbf{x}_{2}$ were drawn from a Gaussian distribution (shown in blue in figure $A 1 A$ ); this is termed the Gaussian case. In the second case, $\mathbf{x}_{1}$ and $\mathbf{x}_{2}$ were based on cube of the same Gaussian distributed values. This generates a leptokurtic distribution which is shown in red in Figure A1A and is termed the non-Gaussian case. In both cases, 30s of data were generated for $\mathbf{s}_{1}$ and $\mathbf{s}_{2}$, with a simulated sample rate of $600 \mathrm{~Hz}$.

For both the Gaussian and non-Gaussian cases, following generation of $\mathbf{s}_{1}$ and $\mathbf{s}_{2}$, the simulated leakage was reduced using the univariate leakage reduction method summarised by Equations A6 and A7; thus generating zero linear correlation between $\mathbf{s}_{1 M}$ (the modified version of $\mathbf{s}_{1}$ ) and $\mathbf{s}_{2}$. The signal envelopes were then computed using a Hilbert transform, and correlation between the envelopes computed. The statistical significance of the measured correlation coefficient was tested using the phase randomisation method described in section 2.4 above. For both the Gaussian and non-Gaussian cases, 5000 iterations of the simulation were run, and the number of false positives counted. These false positive counts are plotted in Figures A1B and A1C for the Gaussian and nonGaussian cases respectively. The associated expected false positive rates are shown by the red line.

Note that, for the Gaussian case, the false positive count in the simulation is in close agreement with that expected. However, in the non-Gaussian case, the false positive count is much higher, showing clearly that leakage reduction only works for Gaussian distributed data. This is a key point and should be borne in mind in any future studies employing this method. MEG data are most often modelled as Gaussian distributed; this is the basis of most analysis techniques (including beamforming). Furthermore, the empirical results gained across previous papers (Brookes et al., 
2012b, Hipp et al., 2012) show the effectiveness of the leakage reduction technique. However, only in the case of genuinely Gaussian distributed data can we assume that the leakage reduction technique offers perfect correction for leakage, and will not result in false positives. Given that there may be deviations from Gaussianity in real MEG data, the leakage reduction algorithm can only be considered a means to supress leakage, rather than correct it completely.
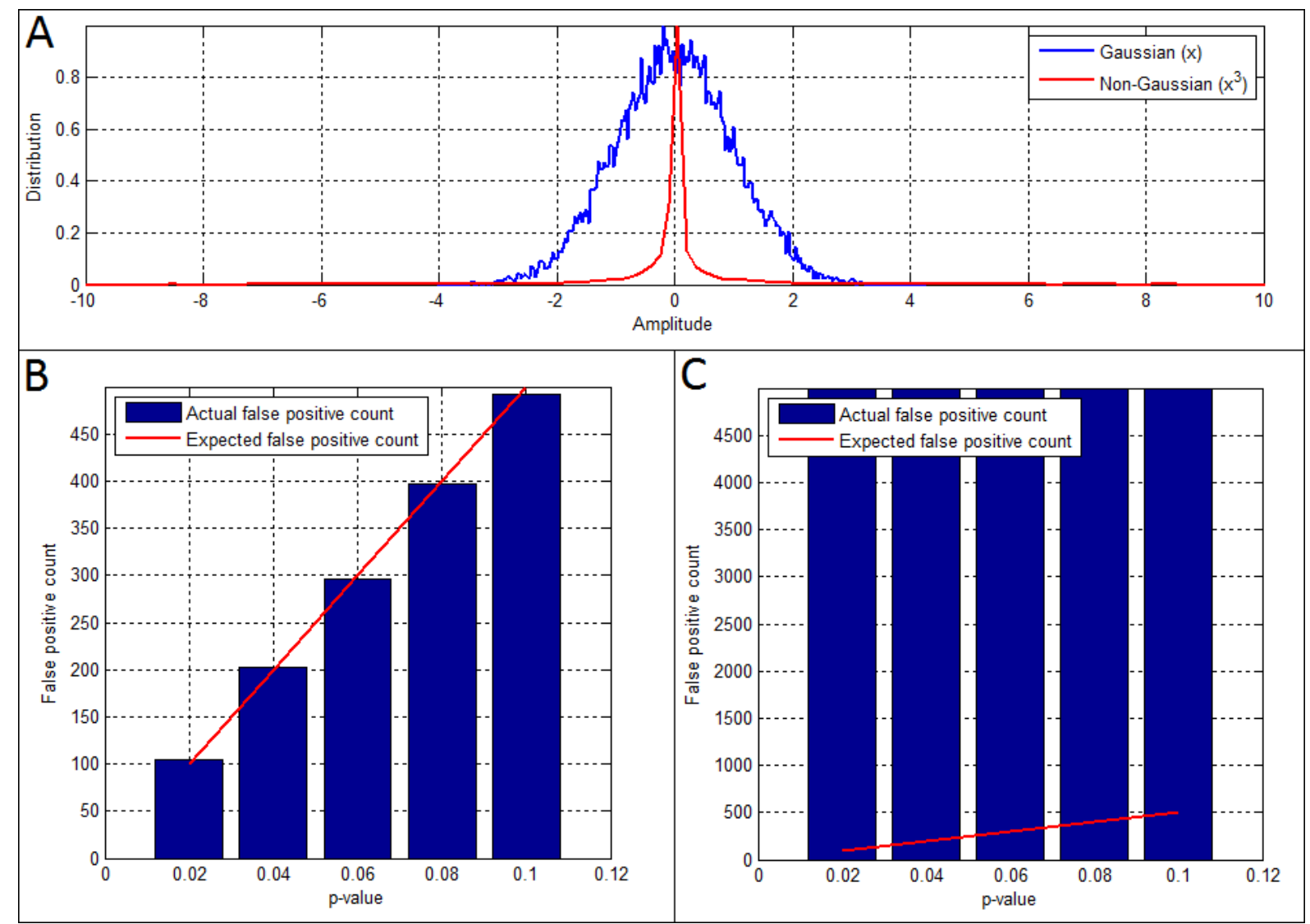

Figure A2:- The effect of non-Gaussian distributed data on false positive rate. A) Shows two separate distributions tested: the blue line shows Gaussian distributed data whereas the red line shows a non-Gaussian (leptokurtic) distribution. B) Shows false positive count plotted against p-value for the Gaussian case. C) Shows false positive count plotted against $p$-value for the non-Gaussian case. In both Figures $B$ and $C$, the expected false positive count is shown in red. Note that false positives are only controlled accurately for Gaussian distributed data.

\section{3) Other considerations}

In addition to the two key points mad above, there are other limitations of leakage suppression via regression which should be elucidated. Firstly, leakage suppression comes at the expense of the loss of any genuine zero phase lag interactions between the seed and test clusters; this may be problematic in cases where, for example, a single (e.g. thalamic) source drives two cortical sources with zero-phase-lag. Secondly, the assumptions of constant leakage across all frequency and all time 
may be invalidated. Meaning that it is possible for the presence of true (zero-lag) physiological interactions within some specific frequency band to generate a misleading picture of the relative amounts of connectivity present within other frequency bands. For example, consider the case where there is a genuine consistent neurophysiological zero lag interaction at $10 \mathrm{~Hz}$ between two sources. Any leakage correction based on a narrow band of frequencies (say $5-20 \mathrm{~Hz}$ ) will make an erroneous overestimate of $\beta$; this will mean that at other frequencies (besides $10 \mathrm{~Hz}$ ) apparent power couplings (due to uncorrected leakage effects) may result. It is also possible that the assumption of constant leakage in time would be invalid in cases where beamformer weighting parameters dynamically change depending on the data (Woolrich et al., 2013). Finally, the approach relies on linear regression and will therefore suffer when the assumptions used to derive these estimators are not met. For example, the estimate of the regression coefficient could be biased by violations of normality (due to outliers for example) in which case alternative regression methods (e.g. robust fitting) may be used. There is also a phenomenon known as regression dilution, in which large variance in the predictor variable itself (in this case the seed voxel cluster) will cause the estimator of the regression coefficient to tend towards zero. This could happen due to external artefacts (like power line noise) which have not been supressed by the inversion procedure. Empirically we have not observed this, however it could become a problem using algorithms without the artefact immunity of the beamformer.

\section{8) ACKNOWLEDGEMENTS:}

We thank the Leverhulme Trust for an Early Career Fellowship awarded to MJB. We also gratefully acknowledge the Medical Research Council, the Wellcome Trust and The University of Nottingham for financial support.

\section{9) REFERENCES:}

Baker A, Luckhoo H, Brookes M, Smith P, Woolrich M, . (2012) Investigating the temporal dynamics of resting state brain connectivity using magnetoencephalography. In: Organisation for Human Brain Mapping Beijing.

Barnes GR, Litvak V, Brookes MJ, Friston KJ (2011) Controlling false positive rates in massmultivariate tests for electromagnetic responses. Neurolmage 1072-1081.

Bassett DS, Meyer-Lindenberg A, Achard S, Duke T, Bullmore E (2006) Adaptive reconfiguration of fractal small-world human brain functional networks. Proceedings of the National Academy of Sciences of the United States of America 103:19518-19523.

Beckmann CF, De Luca M, Devlin JT, Smith SM (2005) Investigations into resting-state connectivity using independent component analysis. . Philos Trans R Soc London 360:1001-1013. 
Biswal B, Yetkin FZ, Haughton VM, Hyde JS (1995) Functional connectivity in the motor cortex of resting human brain using echo planar MRI. Magnetic Resonance in Medicine 34:537-541.

Breakspear M, Terry J, Friston $K$ (2003) Modulation of excitatory synaptic coupling facilitates synchronization and complex dynamics in a nonlinear model of neuronal dynamics. Neurocomputing 52-54:151 - 158.

Breakspear M, Williams LM, Stam CJ (2004) Topographic analysis of phase dynamics in neural systems reveals formation and dissolution of 'dynamic cell assemblies'. Journal of Computational Neuroscience 16:49-68.

Brookes MJ, Hale JR, Zumer JM, Stevenson CM, Francis ST, Barnes GR, Owen JP, Morris PG, Nagarajan SS (2011a) Measuring Functional Connectivity Using MEG: Methodology and Comparison With fcMRI Neurolmage 56:1082-1104.

Brookes MJ, Liddle EB, Hale JR, Woolrich MW, Luckhoo H, Liddle PF, Morris PG (2012a) Task induced modulation of neural oscillations in electrophysiological brain networks. Neurolmage 63:1918-1930.

Brookes MJ, Vrba J, Robinson SE, Stevenson CM, Peters AP, Barnes GR, Hillebrand A, Morris PG (2008) Optimising experimental design for MEG beamformer imaging Neurolmage 39:1788-1802.

Brookes MJ, Woolrich M, Luckhoo H, Price D, Hale JR, Stephenson MC, Barnes GR, Smith SM, Morris $P G$ (2011b) Investigating the Electrophysiological Basis of Resting State Networks using Magnetoencephalography. Proceedings of the National Academy of Science USA 108:16783-16788.

Brookes MJ, Woolrich MW, Barnes GR (2012b) Measuring Functional Connectivity in MEG: a MultiVariate Approach insensitive to linear Source Leakage. Neurolmage 63:910-920.

Chang C, Glover GH (2010) Time-frequency dynamics of resting-state brain connectivity measured with fMRI. Neurolmage 50:81-98.

de Pasquale F, Della Penna S, Snyder AZ, Lewis C, Mantini D, Marzetti A, Belardinelli P, Ciancetta L, Pizzella V, Romani GL, Corbetta M (2010) Temporal dynamics of spontaneous MEG activity in brain networks. Proceedings of the National Academy of Science USA 107:6040-6045.

de Pasquale F, Della Penna S, Snyder AZ, Marzetti L, Pizzella V, Romani GL, Corbetta M (2012) A cortical core for dynamic integration of functional networks in the resting human brain. Neuron 74:753-764. .

Deco G, Corbetta M (2011) The dynamical balance of the brain at rest. Neuroscientist 17:107-123.

Deco G, Jirsa V, McIntosh AR, Sporns O, Kotter $R$ (2009) Key role of coupling, delay, and noise in resting brain fluctuations. Proceedings of the National Academy of Sciences of the United States of America 106:10302-10307.

Fox MD, Raichle ME (2007) Spontaneous fluctuations in brain activity observed with functional magnetic resonance imaging. Nature Reviews Neuroscience 8:700-711.

Fox MD, Snyder AZ, Vincent JL, Corbetta M, Van Essen DC, Raichle ME (2005) The human brain is intrinsically organized into dynamic, anticorrelated functional networks. Proceedings of the National Academy of Sciences of the United States of America 102:9673-9678.

Friston K (1997) Transients metastability and neuronal dynamics. Neurolmage 5:164-171. 
Friston $K$ (2000) The labile brain. I. Neuronal transients and nonlinear coupling. Phil Trans $R$ Soc Lond B 355:215-236.

Ghosh A, Rho Y, McIntosh AR, Kotter R, Jirsa VK, () Noise during rest enables the exploration of the brain's dynamic repertoire. PLOS Comput Biol 4:e1000196. (2008) Noise during rest enables the exploration of the brain's dynamic repertoire. PLoS Computational Biology 4:e1000196. doi:1000110.1001371/journal.pcbi.1000196.

Gross J, Kujala J, Hamalainen M, Timmermann L, Schnitzler A, Salmelin R (2001) Dynamic imaging of coherent sources: Studying neural interactions in the human brain. Proceedings of the National Academy of Science USA 98:694-699.

Hall EL, Woolrich MW, Thomaz CE, Morris PG, Brookes MJ (2013) Using variance information in magnetoencephalography measures of functional connectivity. Neurolmage 67:203-212.

Hipp JF, Hawellek DJ, Corbetta M, Siegel M, Engel AK (2012) Large-scale cortical correlation structure of spontaneous oscillatory activity. Nature Neuroscience 15:884-890.

Honey C, Kotter R, Breakspear M, Sporns $O$ (2007) Network structure of cerebral cortex shapes functional connectivity on multiple time scales. Proceedings of the National Academy of Sciences of the United States of America 104:10240-10245.

Huang MX, Mosher, J.C., Leahy, R.M. (1999) A sensor-weighted overlapping-sphere head model and exhaustive head model comparison for MEG. Physics in Medicine and Biology 44:423-440.

Liu Z, Fukunaga M, de Zwart JA, Duyn JH (2010) Large-scale spontaneous fluctuations and correlations in brain electrical activity observed with magnetoencephalography Neurolmage 102111.

Luckhoo H, Hale JR, Stokes MG, Morris PG, Brookes MJ, Woolrich MW (2012) Inferring task-related networks using Independent Component Analysis in magnetoencephalography Neurolmage 62:530541.

Mantini D, Perucci MG, Del Gratta C, Romani GL, Corbetta M (2007) Electrophysiological signatures of resting state networks in the human brain Proceedings of the National Academy of Science USA 104:13170-13175.

Marzetti L, Della Penna S, Snyder AZ, Pizzella V, Nolte G, de Pasquale F, Romani GL, Corbetta M (2013) Frequency specific interactions of MEG resting state activity within and across brain networks as revealed by the Multivariate Interaction Measure. Neurolmage In Submission.

Nolte G, Bai O, Wheaton L, Mari Z, Vorbach S, Hallett M (2004) Identifying true brain interaction from EEG data using the imaginary part of coherency. Clinical Neurophysiology 115:2292 - 2307.

Prichard D (1994) Generating Surrogate Data for Time Series with Several Simultaneously Measured Variables. Physical Review Letters 73:195-194.

Raichle ME (2009) A Paradigm Shift in Functional Brain Imaging. Journal of neuroscience 29:12729 12734.

Robinson S, Vrba J (1998) Functional Neuroimaging by synthetic Aperture Magnetometry. In Recent Advances in Biomagnetism, ed Yoshimoto T, Kotani M, Kuriki S, Karibe H, Nakasato N, Tohoku Univ Press, Sendai, Japan 302-305. 
Sanchez-Panchuelo RM, Besle J, Beckett A, Bowtell R, Schluppeck D, Francis S (2012) Within-digit functional parcellation of brodmann areas of the human primary somatosensory cortex using functional magnetic resonance imaging at 7 tesla. Journal of Neuroscience 32:15815-15822.

Sarvas J (1987) Basic mathematical and electromagnetic concepts of the biomagnetic inverse problem. Physics in Medicine and Biology 32:11 - 22.

Schoffelen JM, Gross J (2009) Source connectivity analysis with MEG and EEG. Human Brain Mapping 30:1857-1865.

Scholvinck ML, Leopold DA, Brookes MJ, Khader PH (2013) The contribution of electrophysiology to functional connectivity mapping. Neurolmage IN PRESS.

Sekihara K, Hild KE, Nagarajan SS (2006) A novel adaptive beamformer for MEG source reconstruction effective when large background brain activities exist. IEEE Transactions on biomedical engineering 53:1755-1764.

Sekihara K, Nagarajan SS, Peoppel D, Marantz A, Miyashita Y (2001) Reconstructing Spatio-Temporal Activities of Neural Sources Using an MEG Vector Beamformer Technique. IEEE Transactions on Biomedical Engineering 48:760-771.

Smith SM, Fox PT, Miller KL, Glahn DC, Fox M, Mackaya CE, Filippini N, Watkins KE, Toro R, Laird AR, Beckmanna CF (2009) Correspondence of the brain's functional architecture during activation and rest. Proceedings of the National Academy of Science USA 106:13040-13045.

Smith SM, Miller KL, Moeller S, Xu J, Auerbach EJ, Woolrich MW, Beckmann CF, Jenkinson M, Andersson J, Glasser MF, Van Essen DC, Feinberg DA, Yacoub ES, Ugurbil K (2012) Temporallyindependent functional modes of spontaneous brain activity. Proceedings of the National Academy of Sciences of the United States of America 109:3131-3136.

Soto JL, Pantazis D, Jerbi K, Baillet S, Leahy RM, . : (2010) Canonical correlation analysis applied to functional connectivity in MEG. . IEEE International Symposium on Biomedical Imaging, From Nano to Macro, Rotterdam, The Netherlands.

Soto JLP, Pantazis D, Jerbi K, Lachaux JP, Garnero L, Leahy RM (2009) Detection of Event-Related Modulations of Oscillatory Brain Activity with Multivariate Statistical Analysis of MEG Data Human Brain Mapping 30:1922-1934

Stam CJ, Nolte G, Daffertshofer A (2007) Phase Lag Index: Assessment of Functional Connectivity From Multi Channel EEG and MEG With Diminished Bias From Common Sources. Human Brain Mapping 28:1178 - 1193.

Van Drongelen W, Yuchtman M, Van Veen BD, Van Huffelen AC (1996) A Spatial Filtering Technique to Detect and Localize Multiple Sources in the Brain. Brain Topography 9:39-49.

Van Veen BD, Van Drongelen W, Yuchtman M, Suzuki A (1997) Localisation of brain electrical activity via linearly constrained minimum variance spatial filtering. IEEE Transactions on biomedical engineering 44:867-880.

Woolrich MW, Luckhoo H, Baker A, Mohseni H, Barnes GR, Brookes MJ, Rezek I (2013) Dynamic State Allocation for MEG Source Reconstruction. Neurolmage 77:77-92. 